\title{
Diversity, distribution and ecology of benthic molluscan communities on the Portuguese continental shelf
}

\author{
R. Martins, L. Sampaio, V. Quintino* , A.M. Rodrigues \\ Departamento de Biologia e CESAM, Universidade de Aveiro, 3810-193 Aveiro, Portugal
}

\section{A R T I C L E I N F O}

\section{Article history:}

Received 3 June 2013

Received in revised form 31 October 2013

Accepted 13 November 2013

Available online 23 November 2013

\section{Keywords:}

Iberian Coast

Soft-bottom

Molluscs

Sediment

Depth

Hydrodynamics

\begin{abstract}
A B S T R A C T
The diversity, ecology and distribution patterns of the Portuguese continental shelf malacofauna and its relationship with abiotic factors were studied from samples covering the western and the southern coast. A total of 2544 specimens were identified corresponding to 169 taxa, mostly bivalves (62\% of the total taxa). Abra alba was the most abundant and the most frequent species. The alpha diversity ranged from one species to $21 \mathrm{spp} .0 .1 \mathrm{~m}^{-2}$. The highest abundance and diversity were obtained in coarser sediments. Multivariate analysis based on the abundance data identified five major malacological groups: (a) Angulus pygmaeus and Thracia villosiuscula in the coarser sediments of the western inner and mid shelf; (b) Calyptraea chinensis and Leptochiton cancellatus in the heterogeneous and organically enriched sediments of the southern shelf; (c) Angulus fabula, Spisula subtruncata and Pharus legumen in the near shore exposed fine sands; (d) A. alba in muddy fine sands, mainly in the northwestern shelf and (e) Saccella commutata in the southwestern deeper shelf. The malacofauna could be used as a proxy for the major benthic communities known to occur in this area, except in muddy patches, where molluscs were absent or low abundant. Median grain-size, gravel content, depth and hydrodynamic regime were the environmental factors best related to the malacofauna spatial distribution patterns. This study sets the first record of Astarte borealis, Leptochiton asellus, Mercenaria mercenaria and Montacuta phascolionis in the Portuguese shelf and the most northern limit for Anadara polii, Glycymeris nummaria, and Leptochiton algesirensis along the northwestern shelf. This study also gives new ecological insights for several species, in terms of bathymetric range distribution, as well as habitat type and highlighted the transitional characteristics of the molluscan communities from this particular northeastern Atlantic area where boreal, temperate and subtropical faunas can coexist.
\end{abstract}

(c) 2013 Elsevier B.V. All rights reserved.

\section{Introduction}

Studies of macrofaunal benthic communities are relevant to assess the biodiversity and the environmental status of the marine environment (e.g. Cacabelos et al., 2008; Koulouri et al., 2006). Benthic communities are well characterized in Portuguese lagoons (e.g. Quintino et al., 1987, 1989), estuaries (e.g. Moreira et al., 1993; Rodrigues and Quintino, 1993; Rodrigues et al., 2006, 2011), intertidal rocky shores (e.g. Araújo et al., 2005; Pereira et al., 2006; Saldanha, 1995), submarine canyons (e.g. Cunha et al., 2011; Cúrdia et al., 2004) and the continental shelf (e.g. Freitas et al., 2011; Marques, 1987; Martins et al., 2013a, 2013b, 2013c).

Within the bent1hic community, the phylum Mollusca is one of the most diverse and widespread in the marine environment (Rueda et al., 2009). Several works focused on the study of spatial and temporal malacofauna distribution patterns and used them as a surrogate of the whole benthic community (e.g. Cacabelos et al., 2008; Quintino et al., 1986; Rufino et al., 2008, 2010; Urra et al., 2013; Zenetos, 1996). Coastal molluscs (mostly bivalves) also represent an important economic

\footnotetext{
* Corresponding author. Tel.: + 351234370350.

E-mail address: victor.quintino@ua.pt (V. Quintino).
}

resource, being widely exploited in Portugal (Gaspar et al., 2003). Nearly 1600 marine mollusk species are mentioned for the Portuguese coast (Macedo et al., 1999), but no dedicated studies were carried out regarding the diversity of molluscs of the entire Portuguese shelf. This area has a particular ecological and biogeographic interest (Saldanha, 1995), since it corresponds to the majority of the Western and part of the Southwestern Iberian coast, a region of contact between warmer waters from northern Africa and the Mediterranean Sea and colder waters from the North Atlantic (Fiúza, 1983). The area between the latitudes $38^{\circ}$ and $40^{\circ} \mathrm{N}$ mark the transition between two different climatic marine zones (thus, biogeographically distinct), both through the pre-glacial Pliocene, before 3.0 Ma, as well as at the Present-day (Silva et al., 2006, 2010; Monegatti and Raffi, 2007). Furthermore, the Portuguese shelf is dissected by several canyons, important morphological and sedimentary boundaries (Oliveira et al., 2007), and presents a variety of hydrodynamic conditions (Bettencourt et al., 2004) and sediment types (Martins et al., 2012), which increase the probability of occurrence of different faunas.

The scope of the present study was to characterize the diversity and distribution of molluscan communities on the Portuguese continental shelf, to give new insights about the ecology of some mollusk species and to discuss the relationships between the environmental and biological data and its potential use as a surrogate of the overall fauna. 


\section{Material and methods}

\subsection{Study area}

This study was conducted on the entire Portuguese continental shelf, from Caminha ( $41^{\circ} 51.8^{\prime} \mathrm{N}, 9^{\circ} 15.6^{\prime} \mathrm{W}$ ) to Vila Real Santo António ( $36^{\circ}$ $56.1^{\prime} \mathrm{N}, 7^{\circ} 24.7^{\prime} \mathrm{W}$ ), covering the majority of the Western and the Southwestern Iberia (Fig. 1). A total of 145 sampling sites were positioned in a regular grid of perpendicular lines to the coastline, separated from each other 10 to $15 \mathrm{~km}$ (Fig. 1) and stratified by depth ( $<50 \mathrm{~m}, 50 \mathrm{~m}, 75 \mathrm{~m}$, $100 \mathrm{~m},>100 \mathrm{~m}$ ), ranging from 13 to $195 \mathrm{~m}$ water depth. The sites were distributed over the entire survey area in order to cover as much as possible the whole range of potential benthic habitats. At each site, two $0.1 \mathrm{~m}^{2}$ Smith-McIntyre grab samples were collected, one for macrofauna and the other for baseline sediment analyses (grain-size and total organic matter content (TOM)). The samples for macrofaunal analyses were sieved on board over $1 \mathrm{~mm}$ mesh size and the residue fixed in neutralized formalin (4\%) stained with rose Bengal. The detailed grain-size results and the spatial distribution of superficial sediments were given in Martins et al. (2012). The spatial distribution of the sediment fine content and TOM is shown in Fig. 1. Coarser sediments with less than 5\% fines content were dominant in the northwestern inner and mid shelf sector
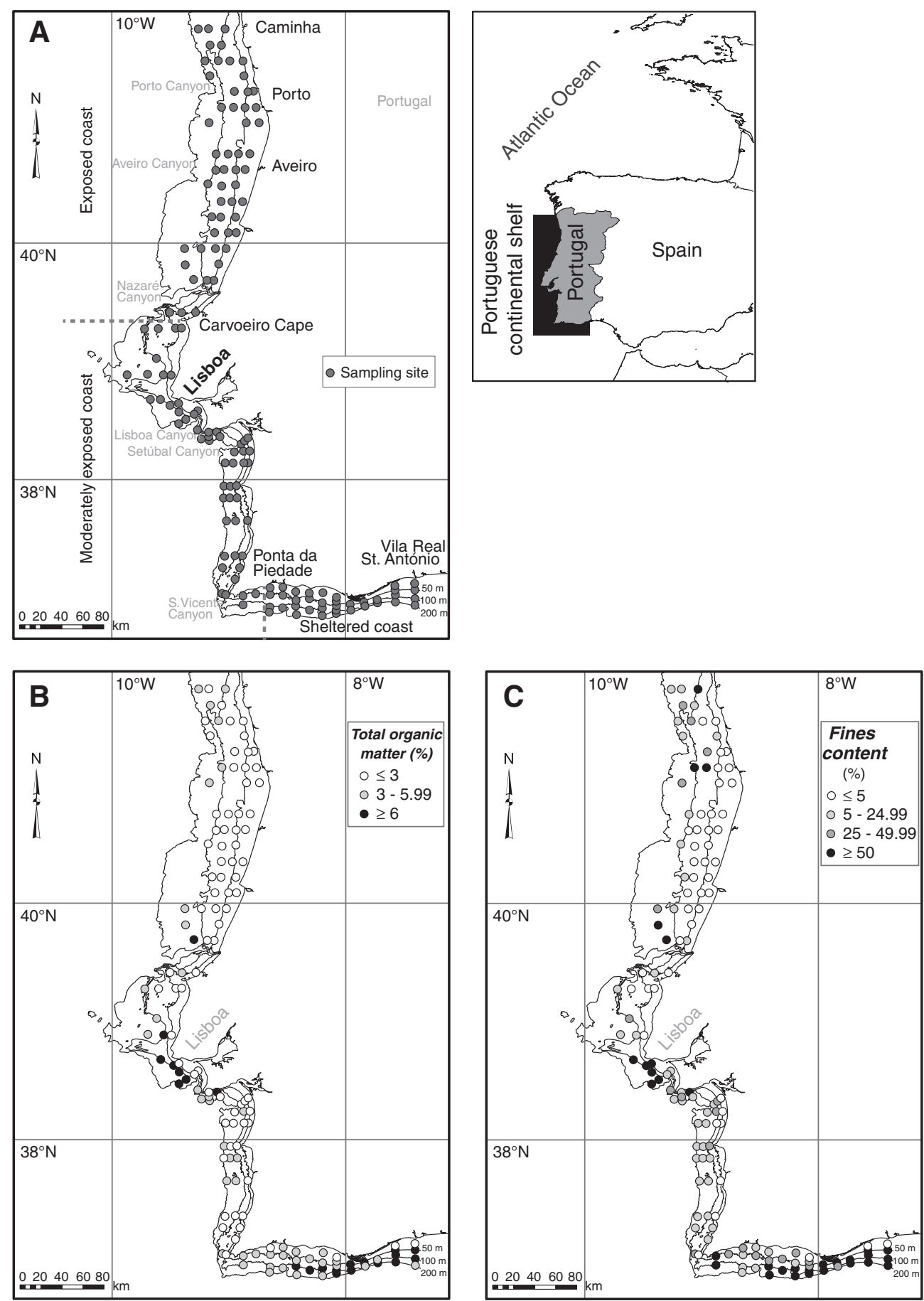

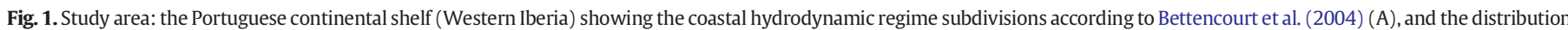
of sediment total organic matter content (B) and fines content (C). 
and immediately south of the Nazaré and Setúbal Canyons. Fine and very fine sands with fine content ranging mainly between 5 and 25\% occurred in the southwestern shelf sector and deeper than $80 \mathrm{~m}$ in the northwestern shelf. In the southern shelf, fine sands with high fines and organic matter content were prevalent in the western part while an extensive mud patch ruled the eastern part. Muddy areas were also identified in the western coast, on the shelves off the Tagus, Douro and Minho rivers. High correlation was found between total organic matter and sediment fines content (Spearman rho $=0.71$ )

\subsection{Laboratory analysis}

In the laboratory, the macrofaunal samples were rinsed with water through a $0.5 \mathrm{~mm}$ mesh sieve, hand sorted and the specimens preserved in ethyl alcohol (70\%). Mollusks were identified to species level, whenever possible, following, namely Graham (1988), Macedo et al. (1999), Tebble (1976), and Thompson (1988). The validity of species name was checked in the WoRMS website (Bouchet and Gofas, 2013) and ecological preferences in those references and others considered suitable (e.g. Antoniadou et al., 2005; Ávila et al., 2008; Bouchet and Gofas, 2013; Duff et al., 2009; Krylova, 2006; Prena et al., 1999; Rodríguez and Sanchéz, 1997).

Abundance, total species richness and alpha diversity (species richness per unit sampling area) were determined per site and mean values were obtained per sediment type, depth classes ( $<30 \mathrm{~m}$; $30-$ $60 \mathrm{~m} ; 60-100 \mathrm{~m} ;>100 \mathrm{~m}$ ), for the western and southern coasts and for the entire coast.

The data matrix with the species abundance per site was square root transformed, and the similarity between sites calculated following the Kulczynski similarity coefficient (K), according to the expression

$K_{(\mathrm{A}, \mathrm{B})}=\frac{1}{2}\left(\frac{\mathrm{W}}{\mathrm{A}}+\frac{\mathrm{W}}{\mathrm{B}}\right)$

where A and B correspond to the total abundance of samples A and B, and $W$ to the sum of the minimum value of abundance of the common species to samples A and B (Legendre and Legendre, 1983). A dummy variable was added in the calculation of the similarity matrix to reduce the influence of samples with very low similarity with the others and therefore increase the redundancy. The resulting similarity matrix was submitted to agglomerative hierarchical clustering, using the unweighted pair-group mean average algorithm (UPGMA) and to ordination analysis using Non-Metric Multidimensional Scaling (NMDS), for the identification of biological affinity groups. These groups were characterized using the mean alpha diversity, total species richness, mean abundance, characteristic species, number of exclusive species and the sediment baseline data. The characteristic species were chosen from the set of species with highest product between constancy (sampling frequency) and fidelity (species preference) per affinity group. Constancy (C) classes were used according to Dajoz (1971) (constant, C > 50.0\%; common, $50.0 \geq C>25.0 \%$; occasional, $25.0 \geq C>12.5 \%$; rare, $C \leq 12.5 \%)$ and fidelity (F) according to Retière (1979) (elective, $F>90.0 \%$; preferential, $90 \geq \mathrm{F}>66.6 \%$; indifferent, $66.6 \geq \mathrm{F}>33.3 \%$; accessory, $33.3 \geq \mathrm{F}>$ $10.0 \%$; accidental, $\mathrm{F} \leq 10.0 \%$ ). The relationship between the environmental and biological data was examined with the BIOENV procedure (BEST routine) based on the environmental variables (depth, median, gravel, sand, fines, biogenic fraction and TOM contents, hydrodynamic regime and latitude). The hydrodynamic regime categories were defined following the classification proposed for the Portuguese coast by Bettencourt et al. (2004); (1 - sheltered Atlantic coast, from Ponta da Piedade to Vila Real de Santo António, most of the southern coast; 2 moderately exposed Atlantic coast, from Cape Carvoeiro to Ponta da Piedade, corresponding mostly to the southwestern coast; 3 - exposed Atlantic coast, from the northern Portuguese border to Cape Carvoeiro, in the northwestern coast).
All the multivariate analyses were performed with PRIMER v.6 (Clarke and Gorley, 2006). Abundance and alpha diversity of each site, abundance of selected species (Abra alba, Corbula gibba, Glycymeris glycymeris, Leptochiton cancellatus, Nassarius reticulatus, Saccella commutata, Thracia villosiuscula and Thyasira flexuosa) and biological affinity groups were plotted in a GIS environment using ArcGis 10 software. The latitudinal distribution of species occurring at least twice in the samples was spatially represented and compared to the literature and worldwide on-line databases (e.g. www.marinespecies.org, www.marbef.org, http://naturalhistory.museumwales.ac.uk/britishbivalves).

\section{Results}

\subsection{Abundance and diversity gradients}

A total of 2544 specimens were analyzed corresponding to 169 molluskan taxa: two Aplocophora, two Scaphopoda, eight Polyplacophora, 53 Gastropoda and 105 Bivalvia. Abundance ranged from one to 172 specimens per $0.1 \mathrm{~m}^{2}$ (Fig. 2-A). From the 145 sites sampled, 13 had no mollusks (Fig. 2-A), similar to the results obtained for Arthropods (crustaceans), but much more than Annelids (one site without specimens; Table 1). Alpha diversity per sampling site ranged from one to 21 molluskan species (Fig. 2-B), which is lower than arthropods or annelids (Table 1). In terms of sediment types, the highest mean abundance and mean alpha diversity was found in gravel (49.6 ind., 11.1 spp. $0.1 \mathrm{~m}^{-2}$ ), the highest total species richness was found in fine sand (89 spp.), while mud presented the lowest values for all measurements (6.7 ind. $0.1 \mathrm{~m}^{-2}, 2.3 \mathrm{spp} .0 .1 \mathrm{~m}^{-2}, 37 \mathrm{spp}$. respectively). Mean alpha diversity, mean abundance and total species richness decreased from shallower to deeper bottoms, peaking between 30 and $60 \mathrm{~m}$ in the case of abundance and total species richness (25.0 ind. $0.1 \mathrm{~m}^{-2}$; $105 \mathrm{spp}$., respectively), probably associated to the prevalence of coarse sediments in the mid-shelf and fine sands in the near shore shelf. The southern shelf presented half of the mean abundance of the western shelf $\left(9.6\right.$ and 20.6 ind. $0.1 \mathrm{~m}^{-2}$, respectively) and lower mean alpha diversity (5.3 and $6.8 \mathrm{spp} .0 .1 \mathrm{~m}^{-2}$, respectively). The most abundant species ( $\mathrm{N}>100$ ind. $0.1 \mathrm{~m}^{-2}$ ) were A. alba, T. villosiuscula, T. flexuosa, Kurtiella bidentata and C. gibba. The most frequent species, occurring at least in 20 sites, were A. alba, C. gibba, T. villosiuscula, S. commutata, Tellina compressa, T. flexuosa, Angulus pygmaeus, Fustiaria rubescens, Gouldia minima, Dosinia lupinus and Tellimya ferruginosa.

\subsection{Habitat preferences and abundance spatial distribution patterns}

Table 2 summarizes the total abundance, total frequency, sediment types and bathymetric ranges of the mollusk species studied within the scope of this work (only taxa identified to species level were considered here). A total of 37 species was recorded exclusively in coarse sediment, mostly in the near shore and mid-shelf (e.g. Digitaria digitaria, G. glycymeris), 33 species in fine sand, mostly from the mid and the deepest shelf (e.g. Bela fuscata, Serratina serrata), three species in mud between 100 and $137 \mathrm{~m}$ (Ecrobia truncata, Nassarius ovoideus, Ringicula buccinea), two species in fine sand and mud from the midshelf (Aporrhais pespelecani, Chaetopleura (Chaetopleura) angulata), and 32 species in both coarse sediment and fine sand in a wide bathymetric range (e.g. Arca tetragona, Clausinella fasciata; Table 2). The remaining species were found in a variety of sediment types and bathymetric range. Some were more frequent and abundant in deeper finer sediments (e.g. T. compressa, S. commutata), others in shallower fine sands (e.g. Angulus fabula, Spisula subtruncata) or in shallower coarse sediments (e.g. Chamelea striatula, Chamelea gallina) (Table 2). A total of 15 species were recorded in maërl in the eastern part of the southern shelf, at $13 \mathrm{~m}$ water depth, being $L$. cancellatus the most abundant. The species Centrocardita aculeata, Chiton (Rhyssoplax) olivaceus, Ocenebra erinaceus 

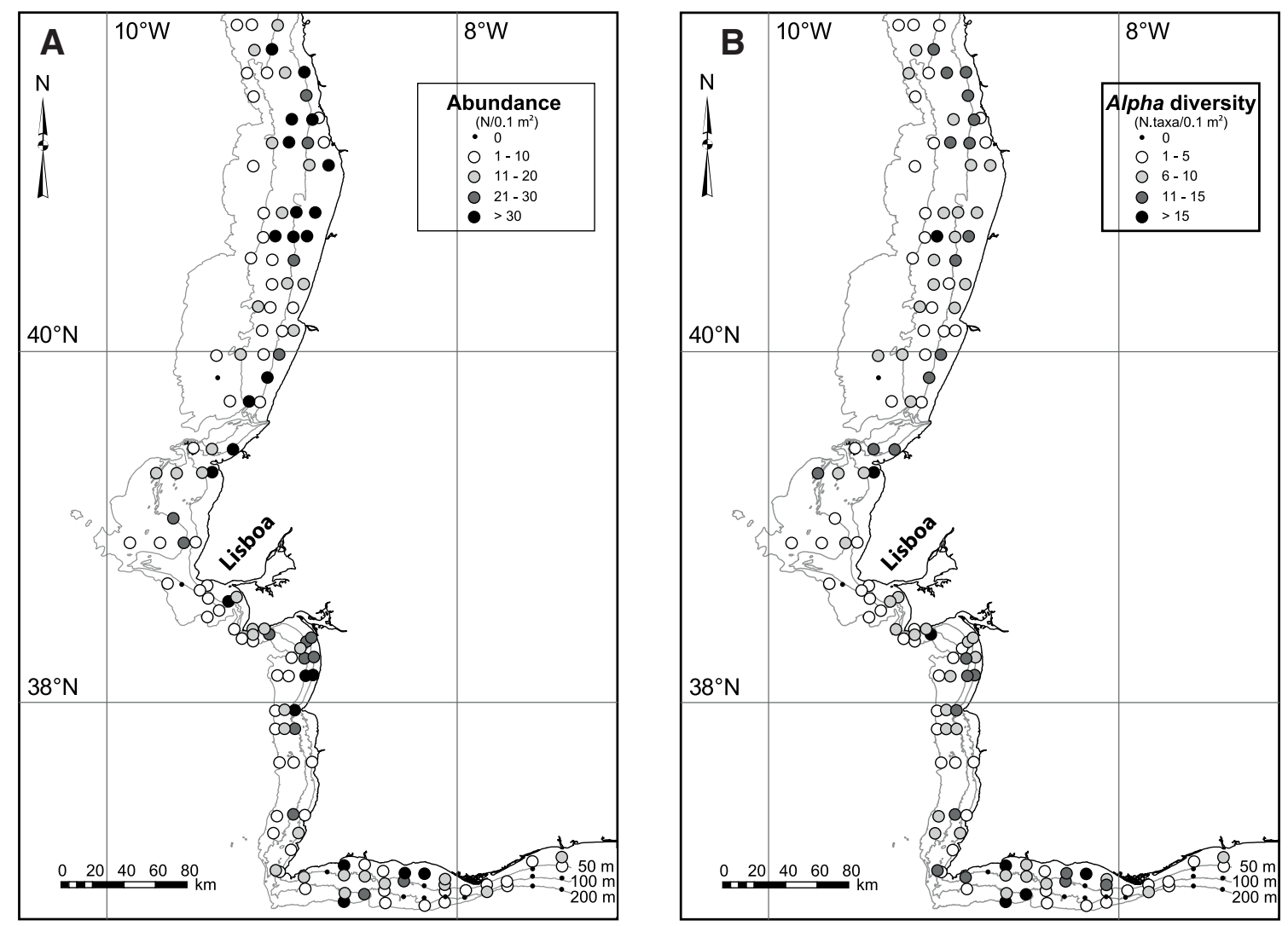

Fig. 2. Spatial distribution of Mollusk abundance (A) and alpha diversity (B) in the Portuguese continental shelf.

and Rocellaria dubia were exclusively recorded in that type of substrate (Table 2).

The latitudinal distribution range of species that occurred at least twice in the samples is shown in Fig. 3. Fig. 4 shows the abundance spatial distribution of selected species. Overall, the majority of the most frequent species occurred along the entire shelf (Fig. 3) apart from A. pygmaeus that occurred exclusively in the coarser sediments of the northwestern shelf and at the head of Setubal canyon, and $T$. ferruginosa only recorded along the western shelf. Species with wider spatial distribution (Fig. 3) showed however higher abundance values in some specific areas: (a) in the northwestern moderately deep fine sediments (e.g. T. flexuosa, Fig. 4-A, A. alba, Fig. 4-B, C. gibba, Fig. 4-C, up to 43,36 and 28 ind. $0.1 \mathrm{~m}^{-2}$, respectively); (b) in the northwestern mid-shelf coarser sediments (e.g. T. villosiuscula, Fig. 4-D, up to 20 ind. $0.1 \mathrm{~m}^{-2}$ ); (c) in the northwestern nearshore shelf (e.g. N. reticulatus, Fig. 4-E, up to eight ind. $0.1 \mathrm{~m}^{-2}$ ); (d) in the

Table 1

Abundance and species richness of the major phyla and the whole benthic macrofauna in the Portuguese continental shelf sediments.

\begin{tabular}{lllll}
\hline & Mollusks & Annelids & $\begin{array}{l}\text { Arthropods } \\
\text { (crustaceans) }\end{array}$ & $\begin{array}{l}\text { Macrofauna } \\
\text { (all) }\end{array}$ \\
\hline Nr. sites with individuals & 132 & 144 & 131 & 145 \\
Nr. sites without individuals & 13 & 1 & 14 & 0 \\
Total abundance & 2544 & 20,241 & 2236 & 30,008 \\
Mean abundance/sample & 19.3 & 140.6 & 17.1 & 207.0 \\
Range of abundance/sample & $1-172$ & $3-670$ & $1-141$ & $7-1307$ \\
Total species richness & 170 & 320 & 195 & 737 \\
$\begin{array}{l}\text { Mean alpha diversity/sample } \\
\text { Range of } \text { alpha diversity/ }\end{array}$ & 7.0 & 29.4 & 6.9 & 45.8 \\
$\quad 1-21$ & $1-66$ & $1-30$ & $3-96$ \\
sample & This & Martins & This study & $\begin{array}{l}\text { Martins } \\
\text { Reference }\end{array}$ \\
& study & et al. & & (2013a) \\
\hline
\end{tabular}

southwestern deepest shelf (e.g. S. commutata, Fig. 4-F, up to 11 ind.0.1 $\mathrm{m}^{-2}$ ). Other species showed more restricted spatial distributions (Fig. 3): (a) exclusively in the northwestern shelf sector, up to Nazaré or Lisbon canyons (e.g. G. glycymeris, Fig. 4-G); (b) in the western shelf sector, from the northern border up to the Setúbal canyon (e.g. D. digitaria); (c) exclusively in the southwestern shelf (e.g. Yoldiella philippiana); (d) from off Peniche to the southern shelf, but more frequent in the southwestern shelf (e.g. Aplocophora sp.); (e) from off Aveiro (e.g. Leptochiton algesirensis, Fig. 4-H) or from off north of Lisbon (e.g. Anadara polii) to the southern shelf (with higher abundance in this sector); (f) from the Setúbal canyon to the southern shelf (e.g. Calyptraea chinensis); (h) exclusively in the southern shelf (e.g. Glans trapezia among other 37 species).

\subsection{Multivariate analysis}

The multivariate analysis of the abundance data is shown in Fig. 5 and the spatial representation of the affinity groups in Fig. 6. Five mollusk communities were identified: A, B1, B2.1, B2.2.1 and B2.2.2 (Figs. 5 and 6). Five sites appeared isolated due to their particular species composition and low abundance. NMDS horizontal dimension (Fig. 5-B) opposed the sites from groups B2.2.1 and B2.2.2 to the sites of group A. Axis 1 was high correlated with fines content, median grain-size, depth, total organic matter content, and gravel content (Spearman rho $=0.60,0.59,0.58,0.58$, and -0.49 , respectively), separating the coarser sediments (group A) from the organically enriched finer sediments (groups B2.2.1 and B2.2.2). NMDS vertical dimension separated B2.1 with B2.2.1 from B1 with B2.2.2. Axis 2 discriminated shallower and other sites located in the northern and exposed shelf sectors, from sites in the southern and sheltered sectors. This axis showed high correlation with hydrodynamic regime areas, latitude and biogenic fraction (Spearman rho $=0.50,0.48,-0.44$ ). The most correlated species (Spearman rho $>0.35$ ) were represented as vectors in Fig. 5. T. villosiuscula and A. pygmaeus were the species most correlated 
Table 2

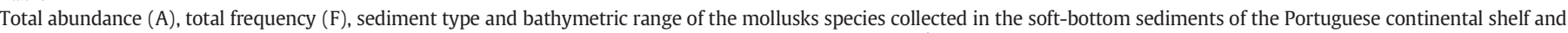

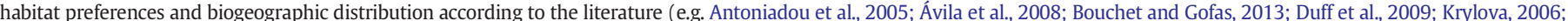

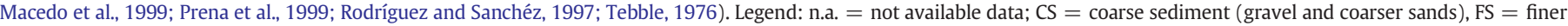

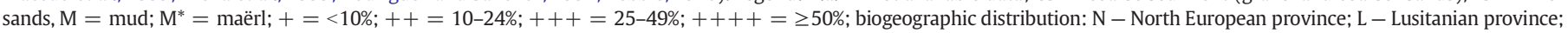

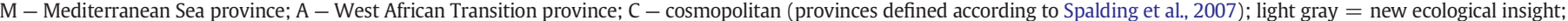
dark gray $=$ new ecological insight for species without available data in the literature.

\begin{tabular}{|c|c|c|c|c|c|c|c|c|c|c|c|c|c|}
\hline \multirow{3}{*}{ Species name } & \multicolumn{7}{|c|}{ This study } & \multicolumn{6}{|l|}{ Literature } \\
\hline & \multirow{2}{*}{ A } & \multirow{2}{*}{$\mathbf{F}$} & \multicolumn{4}{|c|}{ Sedimentary preferences } & \multirow{2}{*}{$\begin{array}{l}\text { Depth } \\
\text { range }\end{array}$} & \multirow{2}{*}{$\begin{array}{c}\text { Habitat preferences } \\
\text { (substrate; maximum depth) }\end{array}$} & & $\log$ & ogra & aph & \\
\hline & & & CS & FS & $\mathrm{M}$ & $\mathrm{M}^{*}$ & & & $\mathrm{~N}$ & $\mathrm{~L}$ & $\mathrm{M}$ & A & $\mathrm{C}$ \\
\hline Abra alba (W. Wood, 1802) & 150 & 37 & ++ & ++++ & + & & $24-135$ & Sand, mud, gravel; 66 m & $\mathrm{X}$ & $\mathrm{X}$ & $\mathrm{X}$ & $\mathrm{X}$ & \\
\hline Abra nitida (Müller, 1776) & 7 & 5 & ++ & ++++ & & & $45-160$ & Muddy sand, mud, gravel; 200 m & $\mathrm{X}$ & $\mathrm{X}$ & $\mathrm{X}$ & & \\
\hline Abra prismatica (Montagu, 1808) & 7 & 5 & ++ & ++++ & & & $74-128$ & Muddy sand, sand; $400 \mathrm{~m}$ & $\mathrm{X}$ & $\mathrm{X}$ & $\mathrm{X}$ & & \\
\hline Acanthochitona fascicularis (Linnaeus, 1767) & 2 & 1 & & & & ++++ & 13 & Attached below stones, sand; $200 \mathrm{~m}$ & $\mathrm{X}$ & $\mathrm{X}$ & $\mathrm{X}$ & & \\
\hline Acteon tornatilis (Linnaeus, 1758 ) & 1 & 1 & ++++ & & & & 47 & Clean fine sand; $250 \mathrm{~m}$ & $\mathrm{X}$ & $\mathrm{X}$ & $\mathrm{X}$ & & \\
\hline Aequipecten opercularis (Linnaeus, 1758) & 3 & 2 & ++++ & & & & $43-76$ & Sand, muddy sand, detritus, gravel; $2660 \mathrm{~m}$ & $\mathrm{X}$ & $\mathrm{X}$ & $\mathrm{X}$ & & \\
\hline Anadara polii (Mayer, 1868) & 7 & 7 & + & +++ & ++++ & & $53-179$ & Muddy sand; $900 \mathrm{~m}$ & & $\mathrm{X}$ & $\mathrm{X}$ & & \\
\hline Anatoma crispata (Fleming, 1828) & 2 & 1 & ++++ & & & & 168 & Stones, shelly or gravelly bottoms; $1000 \mathrm{~m}$ & $\mathrm{X}$ & $\mathrm{X}$ & $\mathrm{X}$ & & \\
\hline Angulus fabula (Gmelin, 1791) & 31 & 14 & + & ++++ & + & & $24-131$ & Silty sand, sand, muddy sand; $55 \mathrm{~m}$ & $\mathrm{X}$ & $\mathrm{X}$ & $\mathrm{X}$ & & \\
\hline Angulus pygmaeus (Lovén, 1846) & 95 & 24 & ++++ & + & & & $25-190$ & Sand, shelly gravel, dead shells; 150 m & $\mathrm{X}$ & $\mathrm{X}$ & $\mathrm{X}$ & $\mathrm{X}$ & \\
\hline Anomia ephippium Linnaeus 1758 & 3 & 3 & ++++ & & & & $33-69$ & Stones, shells, other hard substrata; $146 \mathrm{~m}$ & $\mathrm{X}$ & $\mathrm{X}$ & $\mathrm{X}$ & & \\
\hline Antalis vulgaris (da Costa, 1778) & 3 & 3 & +++ & +++ & +++ & & $29-101$ & Sand, mud; 50 m & $\mathrm{X}$ & $\mathrm{X}$ & $\mathrm{X}$ & & \\
\hline Aporrhais pespelecani (Linnaeus, 1758) & 6 & 2 & & ++++ & ++++ & & $53-92$ & Mud, muddy sand; $180 \mathrm{~m}$ & $\mathrm{X}$ & $\mathrm{X}$ & $\mathrm{X}$ & & \\
\hline Arca tetragona Poli, 1795 & 11 & 5 & ++++ & +++ & & & $33-179$ & Hard substrata (crevices, stones); $2700 \mathrm{~m}$ & $\mathrm{X}$ & $\mathrm{X}$ & $\mathrm{X}$ & & \\
\hline Arcopagia crassa (Pennant, 1777) & 2 & 2 & ++++ & ++++ & & & $41-94$ & Coarse or muddy sand, mud, gravel; $150 \mathrm{~m}$ & $\mathrm{X}$ & $\mathrm{X}$ & $\mathrm{X}$ & & \\
\hline Arcopella balaustina (Linnaeus, 1758) & 1 & 1 & & ++++ & & & 93 & Fine sand, mud, gravel; $750 \mathrm{~m}$ & $\mathrm{X}$ & $\mathrm{X}$ & $\mathrm{X}$ & $\mathrm{X}$ & \\
\hline Astarte borealis (Schumacher, 1817) & 2 & 1 & ++++ & & & & 35 & Gravel, sand; $130 \mathrm{~m}$ & $\mathrm{X}$ & $\mathrm{X}$ & $\mathrm{X}$ & $\mathrm{X}$ & $\mathrm{X}$ \\
\hline Astarte sulcata (da Costa, 1778) & 2 & 2 & & ++++ & & & $49-127$ & Sand, mud, muddy gravel; 2525 m & $\mathrm{X}$ & $\mathrm{X}$ & $\mathrm{X}$ & & \\
\hline Atrina fragilis (Pennant, 1777) & 3 & 3 & & ++++ & & & $17-47$ & Mud, sand, fine gravel; $600 \mathrm{~m}$ & $\mathrm{X}$ & $\mathrm{X}$ & $\mathrm{X}$ & & \\
\hline Axinulus croulinensis (Jeffreys, 1847) & 1 & 1 & & ++++ & & & 117 & Sandy mud; $2000 \mathrm{~m}$ & $\mathrm{X}$ & $\mathrm{X}$ & $\mathrm{X}$ & & \\
\hline Bathyarca pectunculoides (Scacchi, 1835) & 10 & 4 & ++++ & ++++ & & & $130-168$ & Stones, gravel; bathyal depths & $\mathrm{X}$ & $\mathrm{X}$ & $\mathrm{X}$ & & \\
\hline Bela brachystoma (Philippi, 1844) & 1 & 1 & & ++++ & & & 62 & Sand, muddy sand; $60 \mathrm{~m}$ & $\mathrm{X}$ & $\mathrm{X}$ & & & \\
\hline Bela decussata (Locard, 1892) & 2 & 2 & & ++++ & & & $33-126$ & Sandy bottoms; infralittoral & $\mathrm{X}$ & $\mathrm{X}$ & $\mathrm{X}$ & & \\
\hline Bela fuscata (Deshayes, 1835) & 9 & 6 & & ++++ & & & $94-160$ & Gravelly bottoms; intertidal & $\mathrm{X}$ & $\mathrm{X}$ & $\mathrm{X}$ & & \\
\hline Bornia geoffroyi (Payraudeau, 1826) & 2 & 1 & ++++ & & & & 14 & n.a.; n.a. & & $\mathrm{X}$ & $\mathrm{X}$ & & \\
\hline Caecum subannulatum de Folin, 1870 & 6 & 3 & ++++ & & & & $55-74$ & Corals and Laminaria substrates; n.a. & & $\mathrm{X}$ & $\mathrm{X}$ & & \\
\hline Calyptraea chinensis (Linnaeus, 1758) & 17 & 11 & + & ++++ & +++ & & $14-129$ & Below stones or shells in sand or mud; $70 \mathrm{~m}$ & $\mathrm{X}$ & $\mathrm{X}$ & $\mathrm{X}$ & $\mathrm{X}$ & $\mathrm{X}$ \\
\hline Cardiomya costellata (Deshayes, 1833) & 9 & 7 & +++ & ++++ & & & $89-179$ & Muddy sand, gravel; $2000 \mathrm{~m}$ & $\mathrm{X}$ & $\mathrm{X}$ & $\mathrm{X}$ & & \\
\hline Centrocardita aculeata (Poli, 1795) & 1 & 1 & & & & ++++ & 13 & Hard substrate, sand, gravel; $200 \mathrm{~m}$ & & $\mathrm{X}$ & $\mathrm{X}$ & & \\
\hline Chaetopleura (C.) angulata (Spengler, 1797) & 2 & 2 & & ++++ & ++++ & & $38-53$ & Hard substrate, stones, mud; $40 \mathrm{~m}$ & & $\mathrm{X}$ & & & \\
\hline Chamelea gallina (Linnaeus, 1758) & 10 & 6 & ++++ & +++ & & & $17-75$ & Clean sand, muddy sand; $20 \mathrm{~m}$ & & $\mathrm{X}$ & $\mathrm{X}$ & & \\
\hline Chamelea striatula (da Costa, 1778) & 25 & 11 & ++++ & +++ & & & $33-99$ & Clean sand, muddy sand; $55 \mathrm{~m}$ & $\mathrm{X}$ & $\mathrm{X}$ & $\mathrm{X}$ & & \\
\hline Chiton (Rhyssoplax) olivaceus Spengler, 1797 & 1 & 1 & & & & ++++ & 12 & Stones with seaweeds; $30 \mathrm{~m}$ & & $\mathrm{X}$ & $\mathrm{X}$ & & \\
\hline Clausinella fasciata (da Costa, 1778) & 21 & 12 & ++++ & +++ & & & $25-127$ & Gravel; $110 \mathrm{~m}$ & $\mathrm{X}$ & $\mathrm{X}$ & $\mathrm{X}$ & & \\
\hline Corbula gibba (Olivi, 1792) & 118 & 36 & +++ & ++++ & ++ & & $14-128$ & Muddy sand, coarse sand; $250 \mathrm{~m}$ & $\mathrm{X}$ & $\mathrm{X}$ & $\mathrm{X}$ & & \\
\hline Crassopleura maravignae (Bivona And., 1838) & 3 & 2 & ++++ & ++++ & & & 38 & Bioclastic gravel; $320 \mathrm{~m}$ & & $\mathrm{X}$ & $\mathrm{X}$ & & \\
\hline Cuspidaria rostrata (Spengler, 1793) & 8 & 5 & & ++++ & & & $62-147$ & Muddy sand, gravel; $3000 \mathrm{~m}$ & $\mathrm{X}$ & $\mathrm{X}$ & $\mathrm{X}$ & $\mathrm{X}$ & $\mathrm{X}$ \\
\hline Cylichna cylindracea (Pennant, 1777) & 27 & 10 & +++ & ++++ & + & & $24-123$ & Fine sand, Laminaria; $740 \mathrm{~m}$ & $\mathrm{X}$ & $\mathrm{X}$ & $\mathrm{X}$ & & \\
\hline Delectopecten vitreus (Gmelin, 1791) & 1 & 1 & ++++ & & & & 168 & Muddy sand, gravel, shells; $3000 \mathrm{~m}$ & $\mathrm{X}$ & $\mathrm{X}$ & $\mathrm{X}$ & & \\
\hline Digitaria digitaria (Linnaeus, 1758) & 53 & 9 & ++++ & & & & $33-99$ & Gravel, gravelly sand; $1200 \mathrm{~m}$ & $\mathrm{X}$ & $\mathrm{X}$ & $\mathrm{X}$ & & \\
\hline Diplodonta rotundata (Montagu, 1803) & 11 & 7 & +++ & +++ & ++ & & $44-179$ & Gravel, muddy sand; 100 m & $\mathrm{X}$ & $\mathrm{X}$ & $\mathrm{X}$ & & \\
\hline Dosinia exoleta (Linnaeus, 1758) & 1 & 1 & ++++ & & & & 45 & Sand, mud, gravel; $70 \mathrm{~m}$ & $\mathrm{X}$ & $\mathrm{X}$ & $\mathrm{X}$ & & \\
\hline Dosinia lupinus (Linnaeus, 1758) & 37 & 21 & ++++ & +++ & + & & $25-128$ & Sand, sandy mud, shelly gravel; $200 \mathrm{~m}$ & $\mathrm{X}$ & $\mathrm{X}$ & $\mathrm{X}$ & & \\
\hline Ecrobia truncata (Vanatta, 1924) & 6 & 1 & & & ++++ & & 100 & n.a.; $1590 \mathrm{~m}$ & $\mathrm{X}$ & $\mathrm{X}$ & $\mathrm{X}$ & & \\
\hline Epilepton clarkiae (Clark W., 1852) & 1 & 1 & & ++++ & & & 114 & n.a.; deep water & $\mathrm{X}$ & & $\mathrm{X}$ & & \\
\hline Ervilia castanea (Montagu, 1803) & 16 & 1 & ++++ & & & & 33 & Sand; $1800 \mathrm{~m}$ & $\mathrm{X}$ & $\mathrm{X}$ & $\mathrm{X}$ & & \\
\hline Eulima glabra (da Costa, 1778) & 6 & 5 & ++ & ++++ & & & $26-123$ & Ectoparasitic on echinoderms; $200 \mathrm{~m}$ & $\mathrm{X}$ & $\mathrm{X}$ & $\mathrm{X}$ & & \\
\hline Euspira fusca (Blainville, 1825) & 1 & 1 & & ++++ & & & 99 & Muddy sand, mud; 165 m & $\mathrm{X}$ & $\mathrm{X}$ & $\mathrm{X}$ & & \\
\hline Euspira nitida (Donovan, 1804) & 52 & 19 & +++ & +++ & + & + & $13-90$ & Sand, gravel; $200 \mathrm{~m}$ & $\mathrm{X}$ & $\mathrm{X}$ & $\mathrm{X}$ & & \\
\hline Flexopecten glaber (Linnaeus, 1758) & 1 & 1 & ++++ & & & & 66 & Sand, mud, debris with seaweeds; $900 \mathrm{~m}$ & $\mathrm{X}$ & $\mathrm{X}$ & $\mathrm{X}$ & & \\
\hline Fusinus rostratus (Olivi, 1792) & 1 & 1 & & ++++ & & & 100 & Sand, mud, maërl; $500 \mathrm{~m}$ & & $\mathrm{X}$ & $\mathrm{X}$ & & \\
\hline Fustiaria rubescens (Deshayes, 1825) & 53 & 23 & +++ & ++++ & + & & $25-190$ & n.a.; Shelf to bathyal & & $\mathrm{X}$ & $\mathrm{X}$ & & \\
\hline Gari costulata (Turton, 1822) & 29 & 12 & ++++ & + & & & $29-99$ & Muddy sand, coarse sand; 1179 m & $\mathrm{X}$ & $\mathrm{X}$ & $\mathrm{X}$ & $\mathrm{X}$ & \\
\hline Gari tellinella (Lamarck, 1818) & 50 & 5 & ++++ & & & & $33-80$ & Coarse or silty sand, gravel, stones; $110 \mathrm{~m}$ & $\mathrm{X}$ & $\mathrm{X}$ & $\mathrm{X}$ & $\mathrm{X}$ & \\
\hline Gibbomodiola adriatica (Lamarck, 1819) & 1 & 1 & ++++ & & & & 14 & Mud; $75 \mathrm{~m}$ & $\mathrm{X}$ & $\mathrm{X}$ & $\mathrm{X}$ & & \\
\hline Gibbula magus (Linnaeus, 1758) & 1 & 1 & ++++ & & & & 14 & Mud, sand, muddy gravel; $70 \mathrm{~m}$ & $\mathrm{X}$ & $\mathrm{X}$ & $\mathrm{X}$ & $\mathrm{X}$ & \\
\hline Gibbula varia (Linnaeus, 1758) & 1 & 1 & ++++ & & & & 43 & Rock; infralittoral & & $\mathrm{X}$ & $\mathrm{X}$ & & \\
\hline Glans trapezia (Linnaeus, 1767) & 1 & 1 & ++++ & & & & 179 & Hard substrate; $73 \mathrm{~m}$ & & $\mathrm{X}$ & $\mathrm{X}$ & & \\
\hline Glycymeris glycymeris (Linnaeus, 1758) & 42 & 12 & ++++ & & & & $29-99$ & Sand, muddy sand, shelly gravel; $100 \mathrm{~m}$ & $\mathrm{X}$ & $\mathrm{X}$ & $\mathrm{X}$ & & \\
\hline Glycymeris nummaria (Linnaeus, 1758) & 3 & 1 & ++++ & & & & 35 & Muddy sand; infralittoral & & $\mathrm{X}$ & $\mathrm{X}$ & & \\
\hline Goodallia triangularis (Montagu, 1803) & 32 & 4 & ++++ & & & & $33-50$ & Sand, mud, fine and shell gravel; $92 \mathrm{~m}$ & $\mathrm{X}$ & $\mathrm{X}$ & $\mathrm{X}$ & & \\
\hline Gouldia minima (Montagu, 1803) & 58 & 22 & ++++ & +++ & & + & $13-160$ & Gravel, fine sand, sandy mud; 200 m & $\mathrm{X}$ & $\mathrm{X}$ & $\mathrm{X}$ & & \\
\hline Hanleya hanleyi (Bean in Thorpe, 1844) & 1 & 1 & & ++++ & & & 49 & Stones, dead shells, corals; 590 m & $\mathrm{X}$ & $\mathrm{X}$ & $\mathrm{X}$ & & \\
\hline Hiatella arctica (Linnaeus, 1767) & 7 & 7 & ++ & ++++ & & & $29-123$ & Crevices, alga holdfasts, shells; 1400 m & $\mathrm{X}$ & $\mathrm{X}$ & $\mathrm{X}$ & & $\mathrm{X}$ \\
\hline Hyala vitrea (Montagu, 1803) & 1 & 1 & ++++ & & & & 169 & Muddy sand; $50 \mathrm{~m}$ & $\mathrm{X}$ & $\mathrm{X}$ & $\mathrm{X}$ & & \\
\hline Hydrobia acuta neglecta Muus, 1963 & 4 & 3 & & ++++ & & & $29-49$ & n.a.; intertidal & $\mathrm{X}$ & $\mathrm{X}$ & $\mathrm{X}$ & & \\
\hline
\end{tabular}


Table 2 (continued)

\begin{tabular}{|c|c|c|c|c|c|c|c|c|c|c|c|c|c|}
\hline Jolya martorelli (Hidalgo, 1878) & 1 & 1 & & ++++ & & & 99 & Mud; $60 \mathrm{~m}$ & & $\mathrm{X}$ & $\mathrm{X}$ & $\mathrm{X}$ & \\
\hline Kellia suborbicularis (Montagu, 1803) & 2 & 2 & ++++ & ++++ & & & $48-179$ & Crevices, shells, muddy gravel; $120 \mathrm{~m}$ & $\mathrm{X}$ & $\mathrm{X}$ & $\mathrm{X}$ & & \\
\hline Kurtiella bidentata (Montagu, 1803) & 122 & 11 & ++ & +++ & ++ & + & $13-62$ & Muddy sand, fine gravel; $100 \mathrm{~m}$ & $\mathrm{X}$ & $\mathrm{X}$ & $\mathrm{X}$ & & \\
\hline Laevicardium crassum (Gmelin, 1791) & 13 & 6 & ++++ & +++ & ++ & & $48-179$ & Muddy sand, sand, shelly gravel; 2195 m & $\mathrm{X}$ & $\mathrm{X}$ & $\mathrm{X}$ & & \\
\hline Leptochiton algesirensis (Capellini, 1859) & 8 & 3 & ++++ & ++++ & & & $13-179$ & Stones; shallow waters & & $\mathrm{X}$ & $\mathrm{X}$ & & \\
\hline Leptochiton alveolus (M. Sars MS, L, 1846) & 1 & 1 & ++++ & & & & 74 & n.a.; $1350 \mathrm{~m}$ & $\mathrm{X}$ & $\mathrm{X}$ & $\mathrm{X}$ & $\mathrm{X}$ & $\mathrm{X}$ \\
\hline Leptochiton asellus (Gmelin, 1791) & 1 & 1 & ++++ & & & & 74 & Hard substrate in muds; $400 \mathrm{~m}$ & $\mathrm{X}$ & $\mathrm{X}$ & $\mathrm{X}$ & & \\
\hline Leptochiton cancellatus (Sowerby, 1840) & 40 & 13 & +++ & +++ & + & + & $13-179$ & Stones, seashells; $920 \mathrm{~m}$ & $\mathrm{X}$ & $\mathrm{X}$ & $\mathrm{X}$ & & \\
\hline Lepton squamosum (Montagu, 1803) & 2 & 1 & ++++ & & & & 44 & Silty sand, fine gravel; $120 \mathrm{~m}$ & $\mathrm{X}$ & $\mathrm{X}$ & $\mathrm{X}$ & & \\
\hline Limaria loscombi (G.B. Sowerby I, 1823) & 1 & 1 & ++++ & & & & 99 & Fine muddy sand or gravel; $950 \mathrm{~m}$ & $\mathrm{X}$ & $\mathrm{X}$ & $\mathrm{X}$ & & \\
\hline Limatula subovata (Monterosato, 1875) & 57 & 7 & ++++ & & & & $33-99$ & Mud, detritus; $2600 \mathrm{~m}$ & $\mathrm{X}$ & $\mathrm{X}$ & $\mathrm{X}$ & & \\
\hline Lioberus agglutinans (Cantraine, 1835) & 1 & 1 & & ++++ & & & 31 & Mud; $60 \mathrm{~m}$ & & $\mathrm{X}$ & $\mathrm{X}$ & $\mathrm{X}$ & \\
\hline Loripes lucinalis (Lamarck, 1818) & 1 & 1 & & ++++ & & & 130 & Mud, sand, fine gravel; $150 \mathrm{~m}$ & $\mathrm{X}$ & $\mathrm{X}$ & $\mathrm{X}$ & $\mathrm{X}$ & \\
\hline Lucinoma borealis (Linnaeus, 1758) & 1 & 1 & & ++++ & & & 42 & Sand, gravel; $1980 \mathrm{~m}$ & $\mathrm{X}$ & $\mathrm{X}$ & $\mathrm{X}$ & & \\
\hline Lyonsia norwegica (Gmelin, 1791) & 1 & 1 & & ++++ & & & 31 & Sand, silty sand; $250 \mathrm{~m}$ & $\mathrm{X}$ & $\mathrm{X}$ & $\mathrm{X}$ & & \\
\hline Macoma balthica (Linnaeus, 1758) & 2 & 1 & & ++++ & & & 29 & Very fine sediments; $140 \mathrm{~m}$ & $\mathrm{X}$ & $\mathrm{X}$ & $\mathrm{X}$ & & $\mathrm{X}$ \\
\hline Mactra stultorum (Linnaeus, 1758) & 3 & 2 & & ++++ & & & $17-32$ & Clean sand; $60 \mathrm{~m}$ & $\mathrm{X}$ & $\mathrm{X}$ & $\mathrm{X}$ & & \\
\hline Melanella doederleini (Brusina, 1886) & 1 & 1 & ++++ & & & & 16 & n.a.; deep waters & & $\mathrm{X}$ & $\mathrm{X}$ & & \\
\hline Melanella frielei (Jordan, 1895) & 3 & 1 & ++++ & & & & 48 & n.a.; $1385 \mathrm{~m}$ & $\mathrm{X}$ & $\mathrm{X}$ & $\mathrm{X}$ & & \\
\hline Melanella polita (Linnaeus, 1758) & 4 & 4 & ++++ & + & & & $38-103$ & Parasite of Holothurians; circalittoral & $\mathrm{X}$ & $\mathrm{X}$ & $\mathrm{X}$ & & \\
\hline Mercenaria mercenaria (Linnaeus, 1758) & 1 & 1 & & ++++ & & & 45 & Mud; $10 \mathrm{~m}$ & $\mathrm{X}$ & $\mathrm{X}$ & $\mathrm{X}$ & & $\mathrm{X}$ \\
\hline Mitrella minor (Scacchi, 1836) & 1 & 1 & & ++++ & & & 117 & n.a.; $120 \mathrm{~m}$ & & $\mathrm{X}$ & $\mathrm{X}$ & & \\
\hline Modiolus barbatus (Linnaeus, 1758) & 3 & 3 & ++++ & +++ & & & $50-117$ & Rocky shores, Laminaria holdfasts; 110 m & $\mathrm{X}$ & $\mathrm{X}$ & $\mathrm{X}$ & $\mathrm{X}$ & \\
\hline Moerella distorta (Poli, 1791) & 3 & 1 & & ++++ & & & 114 & n.a.; $100 \mathrm{~m}$ & $\mathrm{X}$ & $\mathrm{X}$ & $\mathrm{X}$ & & \\
\hline Moerella donacina (Linnaeus, 1758) & 20 & 12 & ++++ & + & & & $29-99$ & Coarse or silty sand, shelly gravel; $800 \mathrm{~m}$ & $\mathrm{X}$ & $\mathrm{X}$ & $\mathrm{X}$ & & \\
\hline Monophorus perversus (Linnaeus, 1758) & 2 & 1 & & ++++ & & & 48 & Between seaweeds; n.a. & $\mathrm{X}$ & $\mathrm{X}$ & $\mathrm{X}$ & & \\
\hline Montacuta phascolionis Dautzenberg \& Fischer, 1925 & 7 & 5 & ++ & ++++ & ++ & & 14-53 & Commensal with sipunculids; n.a. & $\mathrm{X}$ & $\mathrm{X}$ & $\mathrm{X}$ & & \\
\hline Musculus subpictus (Cantraine, 1835) & 3 & 1 & & ++++ & & & 48 & Under stones or Laminaria; $60 \mathrm{~m}$ & $\mathrm{X}$ & $\mathrm{X}$ & $\mathrm{X}$ & & \\
\hline Myrtea spinifera (Montagu, 1803) & 17 & 14 & ++ & ++++ & & & $26-131$ & Muddy sand, sand, mud, gravel; 250 m & $\mathrm{X}$ & $\mathrm{X}$ & $\mathrm{X}$ & & \\
\hline Nassarius elatus (Gould, 1845) & 8 & 4 & & ++ & ++++ & & $98-137$ & Mud; circalittoral & $\mathrm{X}$ & $\mathrm{X}$ & $\mathrm{X}$ & $\mathrm{X}$ & \\
\hline Nassarius incrassatus (Strøm, 1768) & 3 & 2 & & & ++++ & ++++ & $13-110$ & Below stones, crevices; $200 \mathrm{~m}$ & $\mathrm{X}$ & $\mathrm{X}$ & $\mathrm{X}$ & & \\
\hline Nassarius ovoideus (Locard, 1886) & 2 & 1 & & & ++++ & & 100 & Mud, muddy sands; $100 \mathrm{~m}$ & & $\mathrm{X}$ & $\mathrm{X}$ & & \\
\hline Nassarius reticulatus (Linnaeus, 1758) & 28 & 14 & +++ & ++++ & + & & $21-53$ & Soft and rocky substrates, algae; $200 \mathrm{~m}$ & $\mathrm{X}$ & $\mathrm{X}$ & $\mathrm{X}$ & & \\
\hline Nucula nitidosa Winckworth, 1930 & 53 & 19 & ++ & ++++ & + & + & $13-99$ & Silty fine sand; $250 \mathrm{~m}$ & $\mathrm{X}$ & $\mathrm{X}$ & $\mathrm{X}$ & $\mathrm{X}$ & \\
\hline Ocenebra erinaceus (Linnaeus, 1758) & 1 & 1 & & & & ++++ & 13 & Rocky shores, crevices, stones; n.a. & $\mathrm{X}$ & $\mathrm{X}$ & $\mathrm{X}$ & & \\
\hline Palliolum incomparabile (Risso, 1826) & 17 & 8 & ++++ & ++++ & & & $49-127$ & Sand, gravelly mud, algae, corals; 2000 m & $\mathrm{X}$ & $\mathrm{X}$ & $\mathrm{X}$ & $\mathrm{X}$ & \\
\hline Palliolum tigerinum (O. F. Müller, 1776) & 9 & 5 & ++++ & & & & $45-74$ & Coarse or muddy sand, rock; $380 \mathrm{~m}$ & $\mathrm{X}$ & $\mathrm{X}$ & $\mathrm{X}$ & & \\
\hline Pandora inaequivalvis (Linnaeus, 1758) & 2 & 1 & & ++++ & & & 17 & Sand, sandy mud; $20 \mathrm{~m}$ & $\mathrm{X}$ & $\mathrm{X}$ & $\mathrm{X}$ & & \\
\hline Parthenina indistincta (Montagu, 1808) & 1 & 1 & ++++ & & & & 49 & Sand; $100 \mathrm{~m}$ & $\mathrm{X}$ & $\mathrm{X}$ & $\mathrm{X}$ & & \\
\hline Parvicardium minimum (Philippi, 1836) & 1 & 1 & & ++++ & & & 93 & Mud, sand and fine gravel; $160 \mathrm{~m}$ & $\mathrm{X}$ & $\mathrm{X}$ & $\mathrm{X}$ & $\mathrm{X}$ & \\
\hline Parvicardium pinnulatum (Conrad, 1831) & 5 & 3 & +++ & ++++ & & & $94-130$ & Sand, muddy sand, gravel; $110 \mathrm{~m}$ & $\mathrm{X}$ & $\mathrm{X}$ & $\mathrm{X}$ & $\mathrm{X}$ & \\
\hline Parvicardium scabrum (Philippi, 1844) & 11 & 5 & ++ & ++++ & & & $99-147$ & Sand, mud, coarse sand; $700 \mathrm{~m}$ & $\mathrm{X}$ & $\mathrm{X}$ & $\mathrm{X}$ & $\mathrm{X}$ & \\
\hline Pharus legumen (Linnaeus, 1758) & 4 & 4 & +++ & ++++ & & & $17-69$ & Sand, mud; $150 \mathrm{~m}$ & $\mathrm{X}$ & $\mathrm{X}$ & $\mathrm{X}$ & & \\
\hline Phaxas pellucidus (Pennant, 1777) & 50 & 17 & ++ & ++++ & + & & $24-126$ & Sand, muddy sand, mud, gravel; $150 \mathrm{~m}$ & $\mathrm{X}$ & $\mathrm{X}$ & $\mathrm{X}$ & $\mathrm{X}$ & \\
\hline Philine aperta (Linnaeus, 1767) & 5 & 5 & +++ & ++++ & & & $26-117$ & Sand, mud; $500 \mathrm{~m}$ & $\mathrm{X}$ & $\mathrm{X}$ & $\mathrm{X}$ & $\mathrm{X}$ & \\
\hline Philine cf. quadrata (S. Wood, 1839) & 2 & 1 & ++++ & & & & 16 & n.a.; n.a. & $\mathrm{X}$ & $\mathrm{X}$ & $\mathrm{X}$ & & \\
\hline Philine punctata (Adams J., 1800) & 10 & 3 & ++++ & & & & $16-41$ & Silty sand; $240 \mathrm{~m}$ & $\mathrm{X}$ & $\mathrm{X}$ & $\mathrm{X}$ & & \\
\hline Pitar rudis (Poli, 1795) & 9 & 4 & +++ & ++++ & & & $29-94$ & Sand, gravel; $80 \mathrm{~m}$ & $\mathrm{X}$ & $\mathrm{X}$ & $\mathrm{X}$ & & \\
\hline Plagyostila asturiana Fischer P. in Folin, 1872 & 1 & 1 & ++++ & & & & 74 & n.a.; n.a. & & $\mathrm{X}$ & $\mathrm{X}$ & $\mathrm{X}$ & \\
\hline Raphitoma flavida (Monterosato, 1884) & 1 & 1 & ++++ & & & & 43 & Gravel, seaweeds, rocks; n.a. & & $\mathrm{X}$ & $\mathrm{X}$ & $\mathrm{X}$ & \\
\hline Retusa truncatula (Bruguière, 1792) & 4 & 4 & ++++ & +++ & & & $49-179$ & Sand, muddy sand, mud, Laminaria; $50 \mathrm{~m}$ & $\mathrm{X}$ & $\mathrm{X}$ & $\mathrm{X}$ & & \\
\hline Ringicula auriculata (Ménard de la Groye, 1811) & 7 & 1 & & ++++ & & & 94 & n.a.; $40 \mathrm{~m}$ & $\mathrm{X}$ & $\mathrm{X}$ & $\mathrm{X}$ & & \\
\hline Ringicula buccinea (Brocchi, 1814) & 1 & 1 & & & ++++ & & 137 & n.a.; Infralittoral & $\mathrm{X}$ & $\mathrm{X}$ & $\mathrm{X}$ & & \\
\hline Rocellaria dubia (Pennant, 1777) & 1 & 1 & & & & ++++ & 13 & Rock; shelf & $\mathrm{X}$ & $\mathrm{X}$ & $\mathrm{X}$ & & \\
\hline Saccella commutata (Philippi, 1844) & 65 & 29 & ++ & ++++ & ++ & & $89-179$ & n.a.; 544 & $\mathrm{X}$ & $\mathrm{X}$ & $\mathrm{X}$ & & \\
\hline Scrobicularia plana (da Costa, 1778) & 2 & 2 & ++++ & ++++ & & & $33-90$ & Mud; $30 \mathrm{~m}$ & $\mathrm{X}$ & $\mathrm{X}$ & $\mathrm{X}$ & $\mathrm{X}$ & \\
\hline Serratina serrata (Brocchi, 1814) & 6 & 3 & & ++++ & & & $94-99$ & Sand, mud; $300 \mathrm{~m}$ & $\mathrm{X}$ & $\mathrm{X}$ & $\mathrm{X}$ & & \\
\hline Solecurtus scopula (Turton, 1822) & 1 & 1 & ++++ & & & & 47 & Muddy sand or gravel; $110 \mathrm{~m}$ & $\mathrm{X}$ & $\mathrm{X}$ & $\mathrm{X}$ & & \\
\hline Spisula elliptica (Brown, 1827) & 12 & 7 & ++++ & & & & $74-128$ & Sand, gravel, mud; 200 m & $\mathrm{X}$ & $\mathrm{X}$ & $?$ & & \\
\hline Spisula subtruncata (da Costa, 1778) & 44 & 16 & +++ & ++++ & + & + & $13-90$ & Sand, muddy sand; $200 \mathrm{~m}$ & $\mathrm{X}$ & $\mathrm{X}$ & $\mathrm{X}$ & & \\
\hline Tellimya ferruginosa (Montagu, 1808) & 27 & 20 & +++ & ++++ & ++ & & $17-121$ & Commensal with sea urchin in sand; shelf & $\mathrm{X}$ & $\mathrm{X}$ & $\mathrm{X}$ & & \\
\hline Tellina compressa Brocchi, 1814 & 99 & 28 & + & +++++ & ++ & & $35-140$ & Sand, muddy sand; $200 \mathrm{~m}$ & $\mathrm{X}$ & $\mathrm{X}$ & $\mathrm{X}$ & & \\
\hline Thracia phaseolina (Lamarck, 1818) & 2 & 2 & & ++++ & & & $39-97$ & Fine sand, mud, gravel; $55 \mathrm{~m}$ & $\mathrm{X}$ & $\mathrm{X}$ & $\mathrm{X}$ & & \\
\hline Thracia villosiuscula (MacGillivray, 1827) & 145 & 29 & ++++ & + & & + & $13-80$ & Coarse or fine sand, gravel, mud; $55 \mathrm{~m}$ & $\mathrm{X}$ & $\mathrm{X}$ & $\mathrm{X}$ & & \\
\hline Thyasira flexuosa (Montagu, 1803) & 136 & 26 & +++ & ++++ & & & $25-137$ & Muddy sand, mud; $2190 \mathrm{~m}$ & $\mathrm{X}$ & $\mathrm{X}$ & $\mathrm{X}$ & & \\
\hline Thyasira subovata (Jeffreys, 1881) & 3 & 1 & & ++++ & & & 45 & Detritus; n.a. & $\mathrm{X}$ & $\mathrm{X}$ & $\mathrm{X}$ & & \\
\hline Timoclea ovata (Pennant, 1777) & 30 & 18 & ++ & ++++ & + & & $31-182$ & Sand, gravel; $2489 \mathrm{~m}$ & $\mathrm{X}$ & $\mathrm{X}$ & $\mathrm{X}$ & & \\
\hline Tropidomya abbreviata (Forbes, 1843) & 1 & 1 & ++++ & & & & 179 & Muddy sand; $460 \mathrm{~m}$ & $\mathrm{X}$ & $\mathrm{X}$ & $\mathrm{X}$ & & \\
\hline Turritella communis Risso, 1826 & 27 & 15 & +++ & +++ & +++ & & $25-129$ & Sand, muddy sands; $200 \mathrm{~m}$ & $\mathrm{X}$ & $\mathrm{X}$ & $\mathrm{X}$ & & \\
\hline Turritella turbona Monterosato, 1877 & 23 & 10 & +++ & ++++ & + & & $25-121$ & Mud, muddy sand; $200 \mathrm{~m}$ & & $\mathrm{X}$ & $\mathrm{X}$ & & \\
\hline Venerupis corrugata (Gmelin, 1791) & 2 & 1 & & ++++ & & & 31 & Mixed sandy bottoms; sublittoral & $\mathrm{X}$ & $\mathrm{X}$ & $\mathrm{X}$ & & $\mathrm{x}$ \\
\hline Venus casina Linnaeus, 1758 & 8 & 4 & ++++ & ++++ & & & $38-75$ & Sand, muddy sand, gravel; $200 \mathrm{~m}$ & $\mathrm{X}$ & $\mathrm{X}$ & $\mathrm{X}$ & & \\
\hline Vitreolina curva (Monterosato, 1874) & 1 & 1 & & ++++ & & & 117 & n.a.; shelf & & $\mathrm{X}$ & $\mathrm{X}$ & & \\
\hline Yoldiella philippiana (Nyst, 1845) & 5 & 5 & ++ & ++++ & & & $123-195$ & Sand, muddy sand, detritus; $1200 \mathrm{~m}$ & $\mathrm{X}$ & $\mathrm{X}$ & $\mathrm{X}$ & & \\
\hline
\end{tabular}




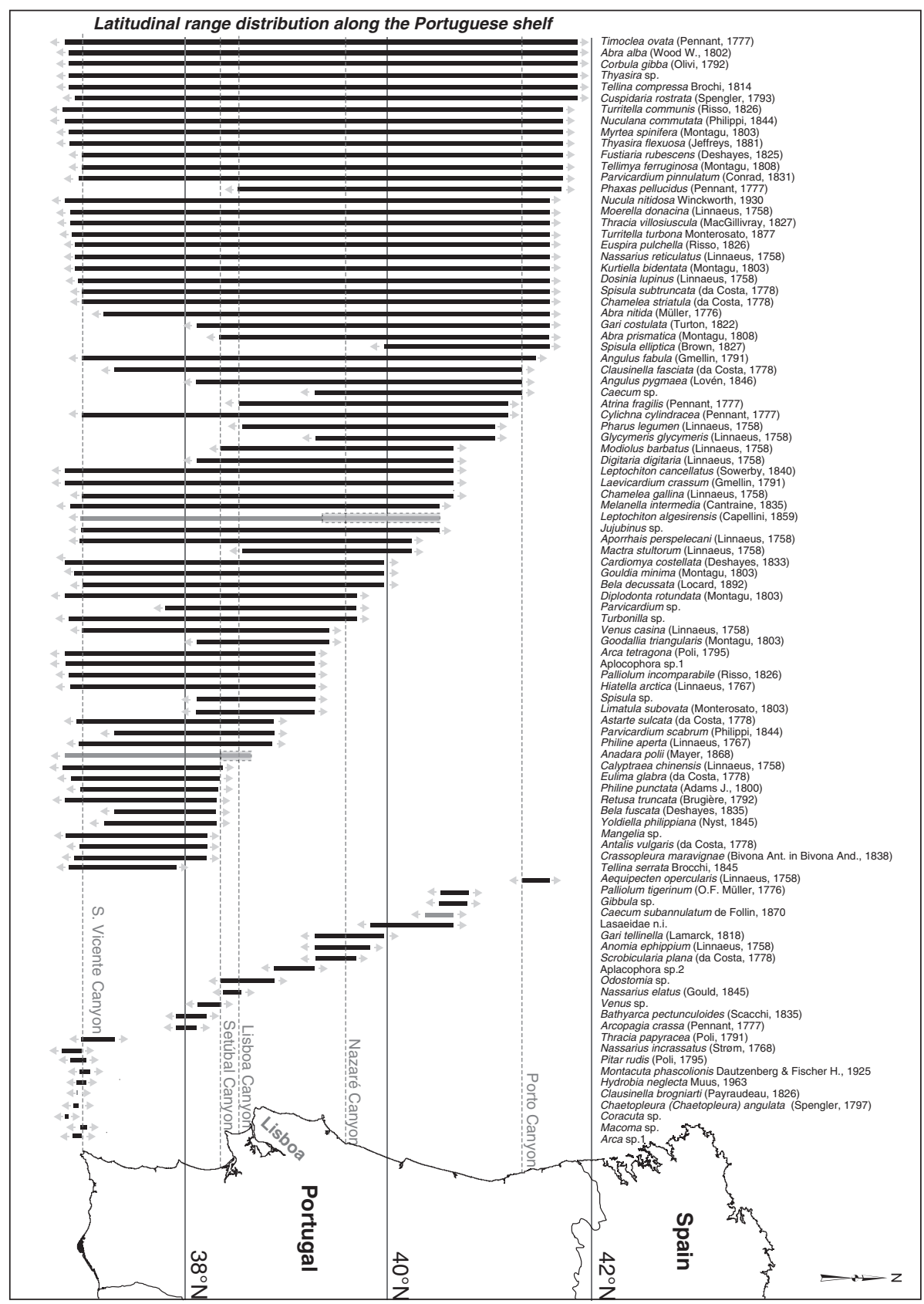

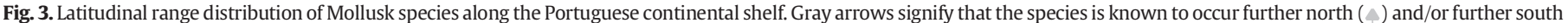
$(\diamond)$. Gray dashed show the proposed northern limit of the worldwide species distribution.

with group A, S. commutata and Aplocophora sp.1 with group B2.2.2, T. compressa, Phaxas pellucidus and A. alba with B2.2.1, A. fabula with B2.1 and C. chinensis with B1.

The environmental and biological characterization of the five mollusk communities is summarized in Tables 3 and 4. Group A included 27 sites, located mainly in areas with coarser sediments of the mid-western shelf (Fig. 6), characterized by the highest gravel and biogenic fraction content (Table 3). This group presented the highest mean species richness and mean abundance. The most abundant species was T. villosiuscula, while the most characteristic was A. pygmaeus (Tables 3 and 4). A total of 31 species were found exclusively here. This group matched community A obtained from the multivariate analysis based on the entire macrofauna dataset and in the polychaetes apart (Table 3 ).

Group B1 gathered 17 sites located on the southern shelf (Fig. 6), mostly mud and fine and very fine sand, characterized by the highest percentage of fines, TOM and biogenic fraction content (Table 3 ).
Mean species richness and mean abundance were moderately high (Table 3). C. chinensis and L. cancellatus were the most characteristic and most abundant species (Tables 3 and 4). The proportion of exclusive species was high (23/67; Table 3$)$. It agreed mostly with group C3 obtained in the macrofauna study and group C2 from the polychaetes study (Table 3 ).

Group B2.1 comprised 13 sites mainly from the near shore shelf (Fig. 6). Sediments were mainly fine sand, with very high content of sand and very low TOM content (Table 3 ). Mean abundance was low and the mean species richness was the lowest. Abra alba was the most abundant and Angulus fabula the most characteristic species (Tables 3 and 4). This group resembled groups B and B1, based on the entire macrofauna and polychaetes only datasets.

Group B2.2.1 corresponded to 38 sites mainly located in the northwestern outer shelf (Fig. 6). Fine and very fine sand and muds were the dominant sediments (Table 3 ). The mean species richness and 

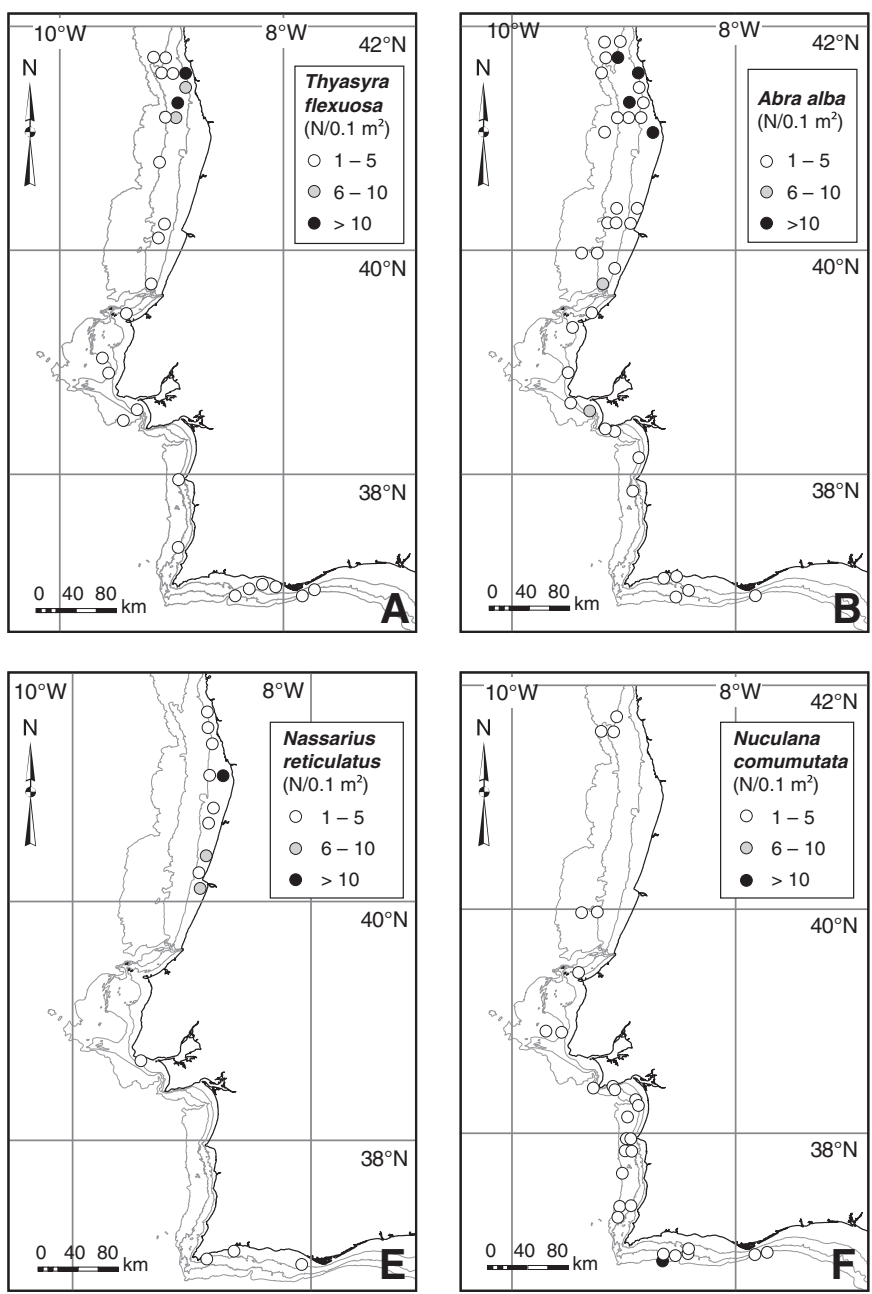
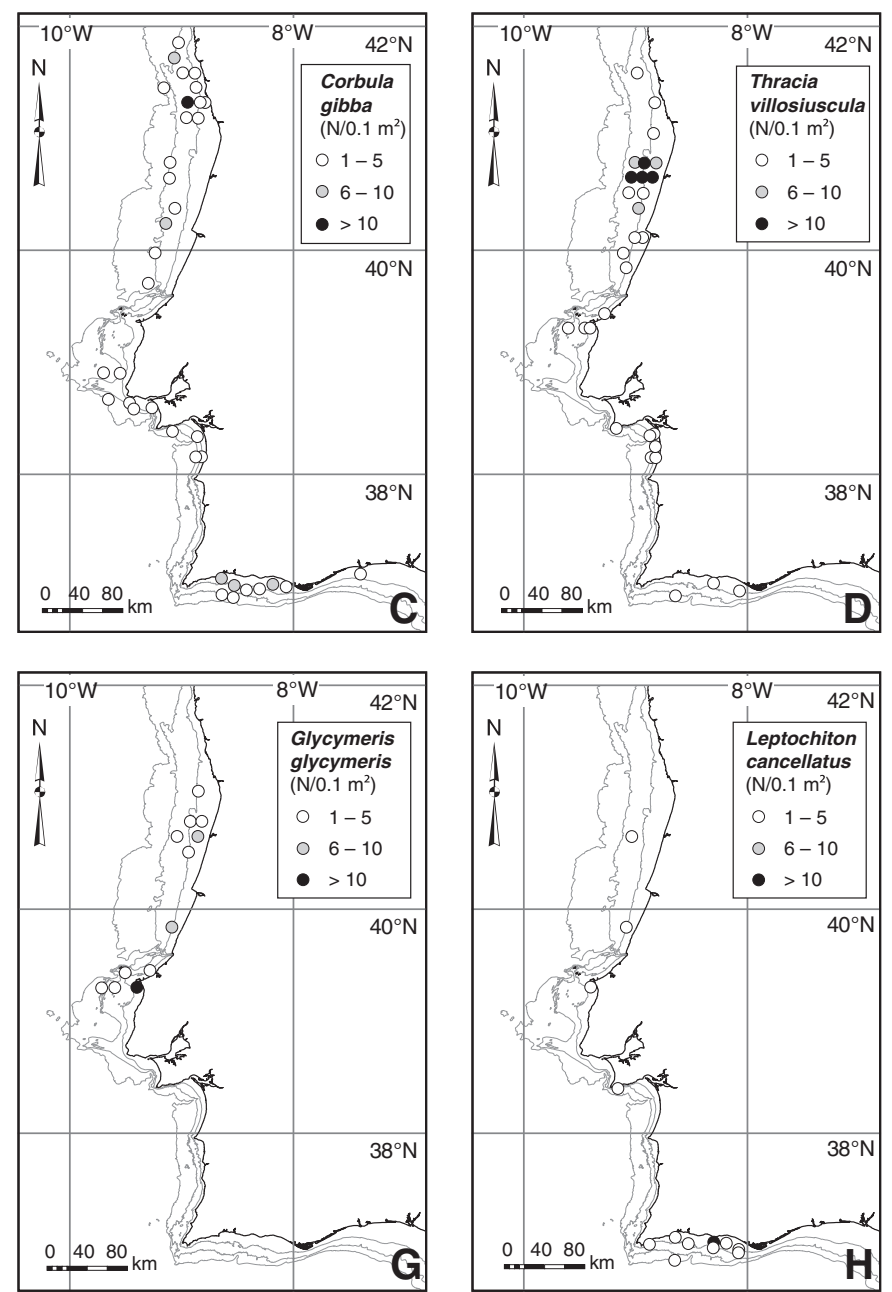

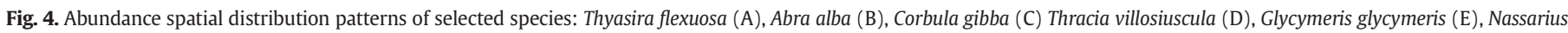
reticulatus (F), Saccella commutata (G) and Leptochiton cancellatus (H).

mean abundance were moderate compared to other groups (Table 3). $T$. flexuosa and $K$. bidentata were the most abundant and T. compressa and A. alba were the most characteristic species. A total of 16 species were exclusively recorded in this group (Table 3 ). This group was close to all muddy sand and muddy affinity groups found among the macrofauna (C1, C2, D and C3) and polychaetes (B2, C1, C3 and C2).

Group B2.2.2 gathered 32 sites, mostly deep and mainly located in the southwestern shelf (Fig. 6). Sediments were similar to group B2.2.1, mainly fine and very fine sand. Mean alpha diversity and mean abundance were moderate. S. commutata was the most abundant and characteristic species (Tables 3 and 4). Nineteen species were exclusively found here (Table 3 ). This community resembled the muddy sand groups $\mathrm{C} 2$ and C1 from macrofauna and polychaetes analyses, respectively.

The environmental variables best related to the biological data were depth, median, gravel content and hydrodynamic regime (BIOENV, rho $=0.319$ ).

\section{Discussion}

\subsection{Diversity and distribution patterns}

The 169 molluskan taxa identified in the course of this work corresponded to $13 \%$ of the Western European malacological fauna (Narayanaswamy et al., 2010). Several studies focused on the molluskan fauna have been carried out in worldwide continental shelves (Table 5). Algae or seagrass meadows, maërl and other hard substrate types tend to be richer in species than soft-bottoms (e.g.Riera et al., 2012; Urra et al., 2013). The lower species richness recorded in those works compared to the present study, may be related to the area sampled, which was much bigger in this work covering six latitudinal degrees. The species Astarte borealis, Leptochiton asellus, Mercenaria mercenaria and Montacuta phascolionis, are here firstly reported for the Portuguese continental shelf (Macedo et al., 1999). This work sets a new meridional distribution limit for A. borealis in the northwestern Portuguese shelf, known before for the Artic and the northern Atlantic and Pacific. Hidalgo (1917) found L. asellus occurring on hard substrates in the Setúbal shore (SW Portugal). Nevertheless, Macedo et al. (1999) state that this species was not yet recorded either in the western or in the southern Portuguese coasts. The North American edible quahog clam M. mercenaria was introduced in Europe in the 19th Century, firstly in the United Kingdom and then all around Europe, from the British Isles to Morocco and the Mediterranean Sea. Macedo et al. (1999) do not recognized this species in the Portuguese fauna, despite the recent release from the Portuguese Government of the checklist of authorized commercial species, which includes this clam species. M. phascolionis is a commensal species well known in northern Europe and in the Mediterranean Sea. In the shallow southern continental shelf it was found in symbiosis with the polychaete Syllis mercedesae and the sipunculid Phascolion (Phascolion) strombus strombus, in empty gastropod shells. This ecological association may be new for science and was reported in Martins et al. (2013a). According to Macedo et al. (1999), A. polii, Glycymeris nummaria and L. algesirensis are southern species with their northern limit located off Setúbal (SW Portugal), off Portimão (S Portugal) and off Carvoeiro Cape (W Portugal), respectively. This study 
A

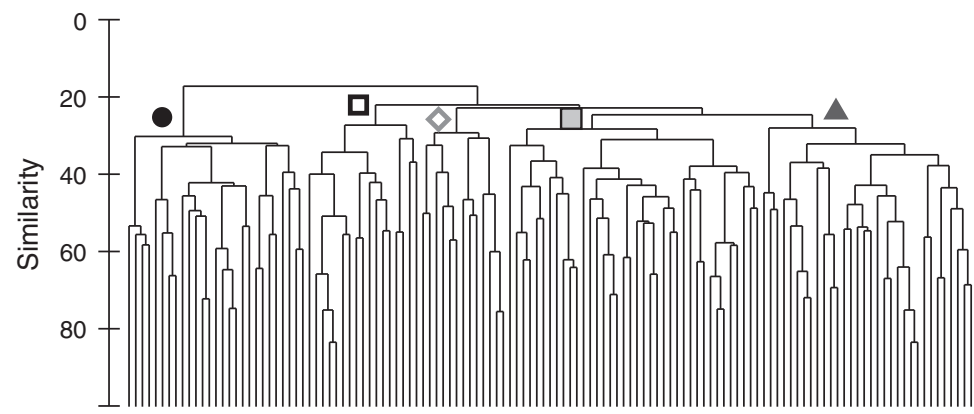

Molluscan affinity groups

- A

口 $B 1$

$\diamond$ B2.1

B $\quad$ B2.2.1

$\Delta \quad$ B2.2.2

100
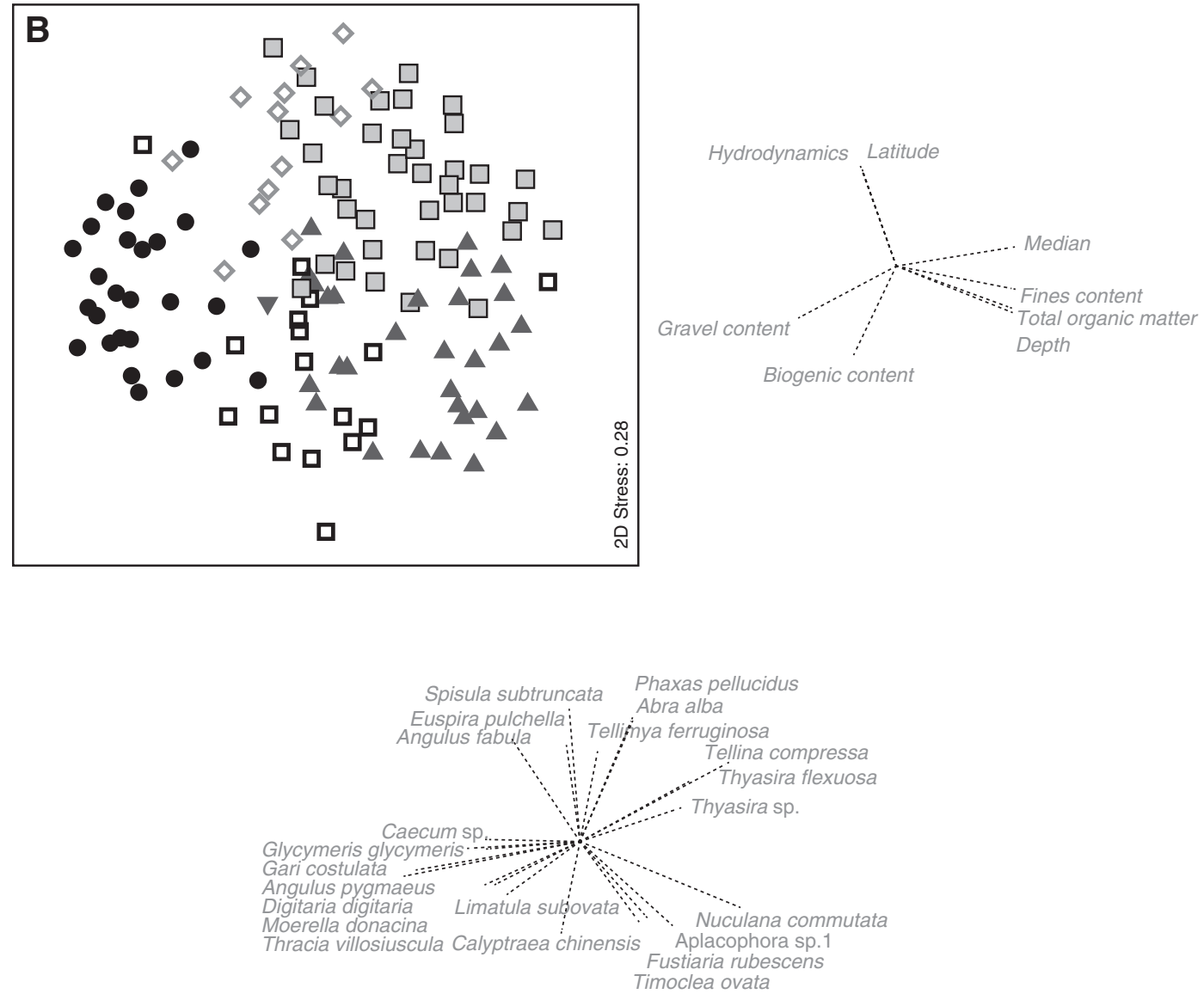

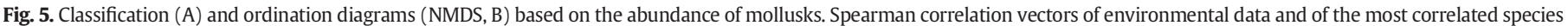
(rho $>0.35)$ are provided as supplementary variables.

shows that these species have wider geographical distributions, being now recorded further north than previously known, in the northwestern shelf. This study also provides new ecological data, such as bathymetric distribution and substrate type preferences, for several species (Table 2). Based in the reports from Graham (1988), Macedo et al. (1999), Tebble (1976), Thompson (1988) among other references, this work sets new maximum depth ranges for 33 species (e.g. A. alba, C. striatula; full list identified as light gray in the depth column in Table 2) and defines bathymetric ranges for eight species, not given in those references (e.g. Caecum subanulatum, M. phascolionis; dark gray in the depth column in Table 2). Regarding species substrate preferences, this study gives new information for 17 species (e.g. Bornia geoffroyi in coarse sediment, Ringicula auriculata in finer sands, $R$. buccinea in mud; dark gray in the sediment types preferences column in Table 2). Sixteen molluskan species which were associated to hard substrate or seaweeds (e.g. Macedo et al., 1999; Tebble, 1976) were here firstly reported in soft bottoms, such as Caecum subannulatum in very coarse sand or
Hanleya hanleyi in very fine sand (light gray in the sediment types preferences column in Table 2). Eulima glabra and Melanella polita, living parasite species of echinoderms (Macedo et al., 1999), were found in coarse sediments and fine sands without echinoderms (Table 2). In several cases, the substrate preference range was widened and now several species are also known to live in mud (Cylichna cylindracea), in finer sand (e.g. Jolya martorelli) or in coarse sediment (e.g. Philine punctata; Table 2). No previously published data existed regarding mollusks inhabiting maërl in the Portuguese territory. From the 15 species found in this study (Table 2), two were reported for this substrate for the first time, namely K. bidentata and C. (Rhyssoplax) olivaceus (e.g. Antoniadou et al., 2005; Grall and Hall-Spencer, 2003; Riera et al., 2012). Maërl beds, which correspond to accumulations of dead and live unattached coralline algae, such as Phymatolithon calcareum, supports a highly diverse community having a relevant ecological and economic importance, namely mollusks, which are commercially exploited (Grall and Hall-Spencer, 2003). As it was recognized in the EU Habitats Directive 


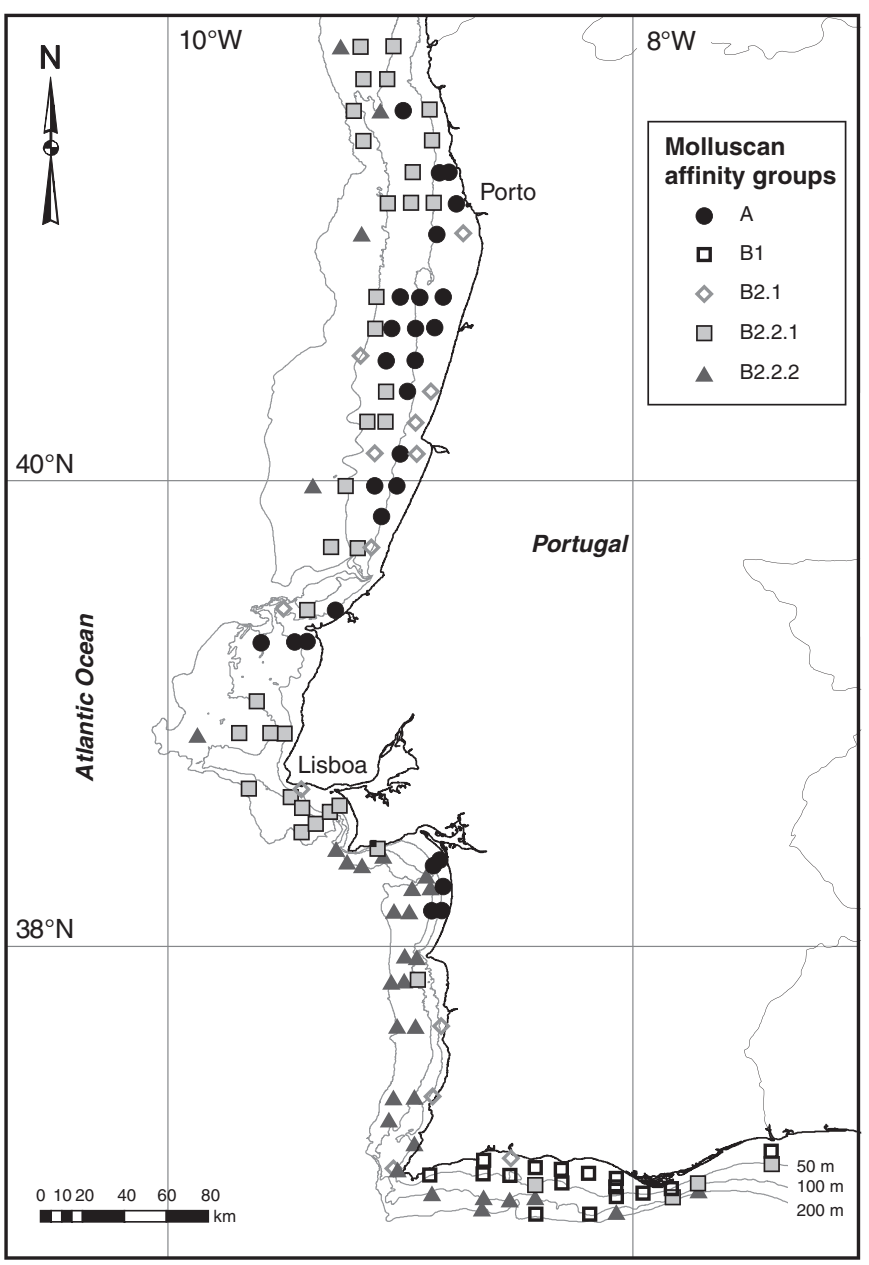

Fig. 6. Spatial distribution of the mollusk communities in the Portuguese continental shelf.

and OSPAR, this is one of the most threatened marine habitats of Europe (Riera et al., 2012).

The highest molluskan diversity and abundance was recorded in the coarse sediments of the western Portuguese shelf. In the northwest (between Porto and south of Aveiro) and the southern Portuguese nearshore coasts, molluskan banks are exploited, mainly the surf clam, Spisula solida (Gaspar et al., 2004, 2005). Other species (e.g. G. glycymeris) are also occasionally taken for the canning industry (Gaspar et al., 2004, 2005). Bivalve dredge fishing is one of the most important fishing activities undertaken in southern Portugal (Pereira et al., 2007). The Portuguese Fisheries Institute regularly assesses the conservation status of the exploited dredge banks (e.g. Gaspar et al., $2004,2005)$, and in the course of this study the following species where identified from the list of the most abundant species occurring there: striped venus ( $C$. gallina and $C$. striatula), the Norwegian egg cockle (Laevicardium crassum), the mature dosinia (Dosinia exoleta), the bean solen (Pharus legumen), the big telina (Arcopagia crassa), the banded venus (C. fasciata) and the dog cockle (G. glycymeris).

\subsection{Benthic macrofauna communities}

This study identified five main molluskan communities. The A. pygmaeus community (group A), recorded in coarser sediments, corresponded to a Lusitanian version of the well-known Venus community (Venus fasciata (now Clausinella) - Spisula elliptica; Thorson, 1957) or to the French Atlantic gravels of Astarte sulcata - Venus casina (Glémarec, 1973). These key northern species showed low abundance in this study, being replaced by others with Lusitanian and/ or Mediterranean biogeographic preferential affinity (e.g. A. pygmaeus, C. subannulatum, C. gallina, Gari costulata, Gari tellinella). This community was identified as the western Portuguese shelf coarse sediments, with Protodorvillea kefersteini, Pisione remota, A. pygmaeus and several other interstitial species (Martins et al., 2013b, 2013c).

The $C$. chinensis and $L$. cancellatus community in mud and finer sands of the southern coast (group B1) showed few resemblances with other communities. The most similar could be a sandy mud circalittoral community described in Italy (shared species: C. chinensis and C. gibba) or the Mediterranean biocenosis of coastal detritic bottoms (shared species: Pitar rudis; Picard, 1965), or the coastal and the offshore detritic biocenosis from the southern Portuguese shelf with $C$. gibba or the infralittoral sands biocenosis with $C$. chinensis, described by Marques (1987). The difficulty in matching our communities with others may be due to the reduced spatial resolution used in this study (a more detailed spatial coverage could change the current image) or to seasonal variations. A southern community was also found in previous studies by Martins et al. (2013b, 2013c).

The A. fabula community (group B2.1), occurring predominantly in fine sand at shallow depth, corresponded to the Northern European community Angulus tenuis and A. fabula (formerly Tellina) defined by Thorson (1957), to the well sorted fine sands from the French Mediterranean coast (Picard, 1965), and to the recently described Portuguese near shore fine sands with Magelona johnstoni, Urothoe pulchella and A. fabula (Martins et al., 2013b, 2013c). This community presented fewer species and specimens than others herein described, possibly due to the strong influence of waves and currents near the coast, which is in agreement with descriptions of equivalent communities elsewhere (Picard, 1965).

The T. compressa and A. alba community in the deep northwestern shelf (group B2.2.1) is widely reported for muddy fine sand habitats in Northern European coasts (Cacabelos et al., 2008; Glémarec, 1973; Moulaert et al., 2007; Thorson, 1957), corresponding to the A. alba (formerly Syndosmya) community. It sets the most southern reference of this community in the northeastern Atlantic (e.g. Cacabelos et al., 2008; Moulaert et al., 2007). This community however presented lower abundance and diversity than similar communities in northern latitudes. It also reflects a mixture of faunal communities already described in this shelf area, one for muds and other for muddy sands (Martins et al., 2013b, 2013c). The overall lower diversity and abundance of mollusks in finer sediment substrates (e.g. Glémarec, 1973; Picard, 1965) could explain this.

The $S$. commutata community in the deeper southwestern and southern shelf (group B2.2.2) was defined by a set of deep and southern species with others spanning a biogeographic distribution from the Boreal to Western African Transition provinces. This may explain why this community could not be easily ascribed to other European communities. This particular community shared the species $S$. commutata and Timoclea ovata with the Atlantic mixed sediments of Nucula nucleus and $P$. rudis of the open sea étage, described by Glémarec (1973) and also shared the species Abra nitida and Y. philippiana with the Maldane sarsi and Ophiura sarsii muddy community of Petersen (1918) and the Mediterranean biocenoses of the terrigenous coastal and deep muds described by Picard (1965). Martins et al. (2013b, 2013c) also identified an community in the muddy sands of the southwestern deep shelf using the whole benthic macrofauna and the polychaetes apart.

This study showed that mollusks may successfully be used to assess the diversity and composition of the major marine benthic communities that may be expected to occur in a particular area, as previously documented by Koulouri et al. (2006) and Zenetos (1996). However, the low abundance, low diversity or even absence of mollusks in most of the muddy areas, oversimplifies the spatial patterns detected from the study of the whole macrofauna, thereby making it advisable that other groups, such as polychaetes, should preferably be included in these assessments (Martins et al., 2013b, 2013c). 
Table 3

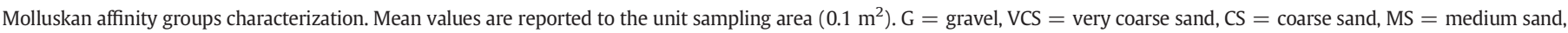

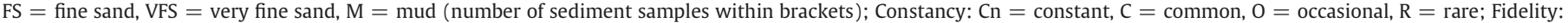
$\mathrm{E}=$ elective, $\mathrm{P}=$ preferential, $\mathrm{I}=$ indifferent, $\mathrm{A}=$ accessory; ${ }^{*}=$ Exclusive species in each group.

\begin{tabular}{|c|c|c|c|c|c|}
\hline Affinity groups & A & B1 & B2.1 & B2.2.1 & B2.2.2 \\
\hline No. of sampling sites & 27 & 17 & 13 & 38 & 32 \\
\hline Main sediment type & Coarser sediments & Finer sands and mud & Fine sand & Finer sands and mud & Finer sands and mud \\
\hline Sites median agreement & $\begin{array}{l}\text { VCS }(12 / 27), \\
\text { G }(8 / 27), \text { CS }(5 / 27), \\
\text { MS }(2 / 27)\end{array}$ & $\begin{array}{l}\text { M (5/17), FS (4/17), } \\
\text { VFS (4/17), VCS (2/17), } \\
\text { CS (1/17), maërl (1/17) }\end{array}$ & $\begin{array}{l}\text { FS (9/13), VCS }(2 / 13), \\
\text { MS (1/13), VFS }(1 / 13)\end{array}$ & $\begin{array}{l}\text { FS }(19 / 38), \text { M }(11 / 38), \\
\text { VFS(5/38), VCS }(1 / 38), \\
\text { CS }(1 / 38), \text { no data }(1 / 38)\end{array}$ & $\begin{array}{l}\text { FS (15/32), VFS ( } 6 / 32), \\
\text { MS ( } 5 / 32), \text { M }(4 / 32), \\
\text { CS ( } 1 / 32), \text { no data }(1 / 32)\end{array}$ \\
\hline Gravel content (mean; \%) & 32.4 & 4.0 & 4.1 & 1.0 & 1.5 \\
\hline Sand conten (mean; \%) & 65.2 & 58.9 & 90.2 & 63.5 & 71.8 \\
\hline Fines content (mean; \%) & 2.3 & 37.0 & 5.7 & 35.7 & 26.7 \\
\hline Biogenic fraction (mean; \%) & 3.8 & 4.8 & 1.0 & 1.2 & 2.2 \\
\hline TOM (mean; \%) & 0.7 & 4.2 & 1.6 & 3.7 & 3.8 \\
\hline Depth (mean; m) & 50.3 & 46.4 & 46.9 & 88.6 & 126.9 \\
\hline Abundance (mean) & 33 & 14 & 12 & 23 & 11 \\
\hline Total species richness & 71 & 59 & 30 & 61 & 86 \\
\hline Mean alpha diversity & 9.1 & 7.7 & 5.2 & 6.5 & 6.1 \\
\hline Nr. of exclusive species & 31 & 22 & 3 & 16 & 19 \\
\hline \multirow{10}{*}{$\begin{array}{l}\text { Characteristic species } \\
\text { (with constancy and } \\
\text { fidelity indications) }\end{array}$} & Angulus pygmaeus (Cn/E) & Calyptraea chinensis $(\mathrm{Cn} / \mathrm{E})^{*}$ & Angulus fabula (Cn/P) & Tellina compressa $(\mathrm{Cn} / \mathrm{I})$ & Saccella commutata $(\mathrm{Cn} / \mathrm{P})$ \\
\hline & Thracia villosiuscula $(\mathrm{Cn} / \mathrm{P})$ & Leptochiton cancellatus $(\mathrm{Cn} / \mathrm{P})$ & Spisula subtruncata $(\mathrm{Cn} / \mathrm{I})$ & Abra alba $(\mathrm{Cn} / \mathrm{I})$ & Aplacophora sp.1 (C/P) \\
\hline & Gari costulata $(\mathrm{C} / \mathrm{E})$ & Montacuta phascolionis (C/E)* & Pharus legumen $(\mathrm{O} / \mathrm{P})$ & Corbula gibba $(\mathrm{Cn} / \mathrm{I})$ & Timoclea ovata $(\mathrm{C} / \mathrm{I})$ \\
\hline & Caecum sp. $(\mathrm{C} / \mathrm{E})^{*}$ & Nucula nitidosa $(\mathrm{C} / \mathrm{I})$ & Cylichna cylindracea $(\mathrm{C} / \mathrm{I})$ & Thyasira sp. (C/P) & Fustiaria rubescens $(\mathrm{C} / \mathrm{I})$ \\
\hline & Glycymeris glycymeris (C/P) & Gouldia mínima (C/I) & Nassarius reticulatus (C/I) & Thyasira flexuosa (C/I) & Parvicardium scabrum $(\mathrm{O} / \mathrm{E})^{*}$ \\
\hline & Digitaria digitaria $(\mathrm{C} / \mathrm{E})^{*}$ & Kurtiella bidentata $(\mathrm{C} / \mathrm{I})$ & Venus casina $(\mathrm{O} / \mathrm{P})$ & Phaxas pellucidus (C/I) & Yoldiella philippiana $(\mathrm{O} / \mathrm{E})^{*}$ \\
\hline & Spisula elliptica $(\mathrm{C} / \mathrm{E})^{*}$ & Hydrobia acuta neglecta $(\mathrm{O} / \mathrm{E})^{*}$ & Turbonilla sp. (O/P) & Tellimya ferruginosa $(\mathrm{C} / \mathrm{I})$ & Cardiomya costellata $(\mathrm{O} / \mathrm{P})$ \\
\hline & Limatula subovata $(\mathrm{O} / \mathrm{E})^{*}$ & Pitar rudis $(\mathrm{O} / \mathrm{P})$ & Philine aperta $(\mathrm{O} / \mathrm{I})$ & Saccella commutata (C/A) & Bela fuscata $(\mathrm{O} / \mathrm{P})$ \\
\hline & Moerella donacina (C/I) & Myrtea spinifera $(\mathrm{C} / \mathrm{I})$ & Phaxas pellucidus (O/I) & Atrina fragilis $(\mathrm{R} / \mathrm{E})^{*}$ & Palliolum incomparabile $(\mathrm{O} / \mathrm{P})$ \\
\hline & Gari tellinella $(\mathrm{O} / \mathrm{E})^{*}$ & $\begin{array}{l}\text { Chaetopleura (Chaetopleura) } \\
\text { angulata }(\mathrm{R} / \mathrm{E})^{*}\end{array}$ & Ensis sp. $(\mathrm{R} / \mathrm{E})^{*}$ & Nassarius elatus (R/E)* & Arca tetragona $(\mathrm{R} / \mathrm{P})$ \\
\hline $\begin{array}{l}\text { Macrofauna affinity } \\
\text { groups agreement } \\
\text { (Martins et al., 2013b) }\end{array}$ & $\mathbf{8 9} \%$ A, $4 \% \mathrm{C} 1,7 \% 0$ & $\mathbf{5 9} \% \mathrm{C} 3,24 \% \mathrm{D}, 18 \% \mathrm{~A}$ & $61 \% B, 23 \% \mathrm{C} 1,8 \% \mathrm{~A}, 8 \% \mathrm{C} 3$ & $\begin{array}{l}\mathbf{5 3 \% C 1}, 18 \% \mathrm{C} 2,18 \% \mathrm{D}, \\
5 \% \mathrm{~B}, 3 \% \mathrm{C} 3,3 \% \mathrm{O}\end{array}$ & 69\%C2, 16\%C1, 6\%B, 6\%D, 3\%A \\
\hline $\begin{array}{l}\text { Polychaetes affinity } \\
\text { groups agreement } \\
\text { (Martins et al., 2013c) }\end{array}$ & $\mathbf{8 5} \%$ A, 7\%B2, 7\%О & $\mathbf{5 3} \% \mathrm{C} 2,29 \% \mathrm{C}, 18 \% \mathrm{~A}, 6 \% \mathrm{C1}$ & 61\%B1, 23\%B2, 8\%A, 8\%C2 & $\begin{array}{l}\mathbf{3 7 \% B 2 ,} \mathbf{3 1} \% \mathrm{C} 1,16 \% \mathrm{C} 3,8 \% \mathrm{~B} 1 \\
3 \% \mathrm{O}, 3 \% \mathrm{C} 2,3 \% \text { no group }\end{array}$ & $\begin{array}{l}\mathbf{6 9} \% \mathrm{C} 1,9 \% \mathrm{C} 2,9 \% \mathrm{C} 3 \\
6 \% \mathrm{~B} 1,3 \% \mathrm{~A}, 3 \% \mathrm{~B} 2\end{array}$ \\
\hline
\end{tabular}

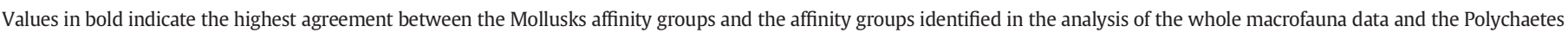
alone. The highest agreement corresponded to the group identified in the coarser sediments.

\subsection{Environmental-biological relationships}

The present study corroborates that the spatial distribution patterns of marine mollusks are influenced by substrate characteristics, depth and hydrodynamics (e.g. Cacabelos et al., 2008; Koulouri et al., 2006; Michel et al., 2011; Quintino et al., 1986; Rufino et al., 2010). It is widely accepted that some organisms are best adapted to specific soft bottom habitats, the species they belong to showing higher abundance and biomass in a particular grain-size range (Hily, 1987). This circumstance also influences their growth rate, burrowing time, foraging effectiveness and metabolic activity (Byrnes et al., 2003; Huz et al., 2002). The variety of the Portuguese shelf sediment types (Martins et al., 2012) supported the high molluskan diversity, some exclusively or abundantly recorded in a specific soft substrate type (e.g. D. digitaria) and others found in a broader substrate range (e.g. A. alba) (e.g. Byrnes et al., 2003; Cacabelos et al., 2008). However, it is recognized that marine biological distribution patterns are also depth related in several continental shelves (Benkendorfer and Soares-Gomes, 2009; Moulaert et al., 2007). This study documented the decrease of abundance and alpha diversity with depth, which can be related to the decrease of the quantity and quality of Chlorophyll a, as it was suggested by Karakassis and Eleftheriou (1997). The occurrence of a fine sands community with fewer species, in shallow waters, may be explained by the harsh hydrodynamic conditions induced by waves and bottom currents rather than the preference for shallower depths (e.g. Glémarec, 1973; Picard, 1965).

Finally, the Portuguese shelf is influenced by a complex current system and seasonal upwelling events that affect productivity and summer and winter Sea Surface Temperatures (SSTs) (Fiúza, 1983; Nascimento et al., 2012). This favors the mixing of subtropical warm and northern cold waters in the Portuguese continental shelf. Colder and nutrientrich waters over the continental shelf during upwelling periods could, at least in part, explain the occurrence at these latitudes of colder water species (e.g. Bathyarca pectunculoides, L. asellus), while higher mean SST in the southern coast, as well as the downwelling events, could explain the record of several warmer water species, particularly in the south (e.g. Lioberus agglutinans, Turritella turbona) (Silva et al., 2006). In fact, SST seems to be a key factor in certain species distribution (Silva et al., 2006, 2010; Raffi et al., 1985). Along the Iberian coasts, the minimal reproduction temperature for some thermophilic gastropod species is $20^{\circ} \mathrm{C}$ (Silva et al., 2010; Raffi et al., 1985). The medium monthly SST is very variable along the Portuguese shelf, reaching $20^{\circ} \mathrm{C}$ in the SW coast (from Nazaré canyon till Cape S. Vincent; $39-$ $37^{\circ} \mathrm{N}$ ). This can contribute to the definition of a transitional faunal break zone, separating northern "Franco-Iberian" colder water communities from southern, "Mediterranean-Moroccan" warmer water communities such was suggested by Silva et al. (2010). Rapid changes in shelf conditions, such as the presence of canyons cannot be discarded in the latitudinal distribution of species (Cunha et al., 2011; Spalding et al., 2007). However, the most relevant shelf morphologies (mainly Nazaré, Lisbon and S. Vicente canyons) are coincident with that transitional faunal shelf zone, being difficult to conclude if those structures affect the mollusks latitudinal distribution along the Portuguese shelf.

\section{Conclusions}

More than 2500 specimens were identified corresponding to 169 taxa, in the Portuguese continental shelf. The present study showed that the most abundant and frequent species was Aa alba. This work sets the first record of Aa borealis, L. asellus, M. mercenaria and $M$. phascolionis in the Portuguese coast and extends the northern limit of A. polii, G. nummaria and L. algesirensis to the northwestern Portuguese shelf. Furthermore, the present study provides new information on 
Table 4

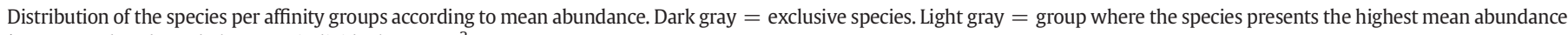
$*=$ Mean abundance below 0.05 individuals $0.1 \mathrm{~m}^{-2}$.

\begin{tabular}{|c|c|c|c|c|c|}
\hline Species & A & B1 & B2.1 & B2.2.1 & $\mathrm{B} 2.2 .2$ \\
\hline Caecum sp. & 2.9 & & & & \\
\hline Limatula subovata (Monterosato, 1875) & 2.0 & & & & \\
\hline Digitaria digitaria (Linnaeus, 1758) & 1.9 & & & & \\
\hline Gari tellinella (Lamarck, 1818) & 1.9 & & & & \\
\hline Goodallia triangularis (Montagu, 1803) & 1.2 & & & & \\
\hline Ervilia castanea (Montagu, 1803) & 0.6 & & & & \\
\hline Gibbula sp. & 0.6 & & & & \\
\hline Spisula elliptica (Brown, 1827) & 0.4 & & & & \\
\hline Palliolum tigerinum (O. F. Müller, 1776) & 0.3 & & & & \\
\hline Philine punctata (Adams J., 1800) & 0.3 & & & & \\
\hline Loripes sp. & 0.3 & & & & \\
\hline Caecum subannulatum de Folin, 1870 & 0.2 & & & & \\
\hline Aequipecten opercularis (Linnaeus, 1758) & 0.1 & & & & \\
\hline Anomia ephippium Linnaeus 1758 & 0.1 & & & & \\
\hline Glycymeris nummaria (Linnaeus, 1758) & 0.1 & & & & \\
\hline Lasaeidae n.i. & 0.1 & & & & \\
\hline Spisula sp. & 0.1 & & & & \\
\hline Tellina distorta Poli, 1791 & 0.1 & & & & \\
\hline Melanella frielei (Jordan, 1895) & 0.1 & & & & \\
\hline Thracia villosiuscula (MacGillivray, 1827) & 5.1 & 0.2 & 0.2 & & * \\
\hline Angulus pygmaeus (Lovén, 1846) & 3.5 & & & & * \\
\hline Glycymeris glycymeris (Linnaeus, 1758) & 1.5 & & 0.1 & & \\
\hline Gouldia minima (Montagu, 1803) & 1.2 & 0.8 & & 0.1 & 0.2 \\
\hline Gari costulata (Turton, 1822) & 1.0 & & & * & \\
\hline Dosinia lupinus (Linnaeus, 1758) & 0.6 & 0.1 & 0.6 & 0.3 & \\
\hline Moerella donacina (Linnaeus, 1758) & 0.6 & 0.1 & 0.1 & & * \\
\hline Clausinella fasciata (da Costa, 1778) & 0.4 & 0.2 & 0.2 & 0.1 & \\
\hline Palliolum incomparabile (Risso, 1826) & 0.3 & & & & 0.3 \\
\hline Chamelea gallina (Linnaeus, 1758) & 0.2 & & 0.1 & 0.1 & \\
\hline Calyptraea chinensis (Linnaeus, 1758) & & 1.0 & & & \\
\hline Montacuta phascolionis Dautzenberg and Fischer H., 1925 & & 0.4 & & & \\
\hline Hydrobia acuta neglecta Muus, 1963 & & 0.2 & & & \\
\hline Nassarius incrassatus (Strøm, 1768) & & 0.2 & & & \\
\hline Musculus subpictus (Cantraine, 1835) & & 0.2 & & & \\
\hline Monophorus perversus (Linnaeus, 1758) & & 0.1 & & & \\
\hline Bornia geoffroyi (Payraudeau, 1826) & & 0.1 & & & \\
\hline Acanthochitona fascicularis (Linnaeus, 1767) & & 0.1 & & & \\
\hline Venerupis corrugata (Gmelin, 1791) & & 0.1 & & & \\
\hline Chaetopleura (Chaetopleura) angulata (Spengler, 1797) & & 0.1 & & & \\
\hline Macoma balthica (Linnaeus, 1758) & & 0.1 & & & \\
\hline Corbula gibba (Olivi, 1792) & 0.4 & 2.1 & & 1.8 & 0.1 \\
\hline Leptochiton cancellatus (Sowerby, 1840) & 0.2 & 1.9 & & & 0.1 \\
\hline Myrtea spinifera (Montagu, 1803) & & 0.4 & 0.2 & 0.1 & 0.1 \\
\hline Leptochiton algesirensis (Capellini, 1859) & * & 0.3 & & & \\
\hline Aporrhais pespelecani (Linnaeus, 1758) & & 0.3 & & * & \\
\hline Pitar rudis (Poli, 1795) & & 0.3 & & & 0.1 \\
\hline Crassopleura maravignae (Bivona Ant. in Bivona And., 1838) & * & 0.1 & & & \\
\hline Antalis vulgaris (da Costa, 1778) & & 0.1 & & & * \\
\hline Hiatella arctica (Linnaeus, 1767) & & 0.1 & & * & 0.1 \\
\hline Abra alba (W. Wood, 1802) & 0.2 & 0.2 & 3.1 & 2.5 & 0.2 \\
\hline Spisula subtruncata (da Costa, 1778) & * & 0.1 & 1.5 & 0.6 & \\
\hline Nassarius reticulatus (Linnaeus, 1758) & 0.2 & 0.2 & 1.4 & 0.1 & \\
\hline Angulus fabula (Gmelin, 1791) & & & 1.1 & 0.4 & 0.1 \\
\hline
\end{tabular}


Table 4 (continued)

Pharus legumen (Linnaeus, 1758)

Venus casina Linnaeus, 1758

Philine aperta (Linnaeus, 1767)

Turbonilla sp.

Nassarius elatus (Gould, 1845)

Ecrobia truncata (Vanatta, 1924)

Mactra sp.

Thyasira flexuosa (Montagu, 1803)

Kurtiella bidentata (Montagu, 1803)

Tellina compressa Brocchi, 1814

Thyasira sp.

Phaxas pellucidus (Pennant, 1777)

Nucula nitidosa Winckworth, 1930

Euspira nitida (Donovan, 1804)

Cylichna cylindracea (Pennant, 1777)

Chamelea striatula (da Costa, 1778)

Tellimya ferruginosa (Montagu, 1808)

Turritella turbona Monterosato, 1877

Turritella communis Risso, 1826

Aplacophora sp.2

Abra prismatica (Montagu, 1808)

Parvicardium scabrum (Philippi, 1844)

Bathyarca pectunculoides (Scacchi, 1835)

Ringicula auriculata (Ménard de la Groye, 1811)

Serratina serrata (Brocchi, 1814)

Yoldiella philippiana (Nyst, 1845)

Venus sp.

Saccella commutata (Philippi, 1844)

Fustiaria rubescens (Deshayes, 1825)

Aplacophora sp.1

Timoclea ovata (Pennant, 1777)

Laevicardium crassum (Gmelin, 1791)

Arca tetragona Poli, 1795

Diplodonta rotundata (Montagu, 1803)

Cardiomya costellata (Deshayes, 1833)

Abra nitida (Müller, 1776)

Anadara polii (Mayer, 1868)

Parvicardium pinnulatum (Conrad, 1831)

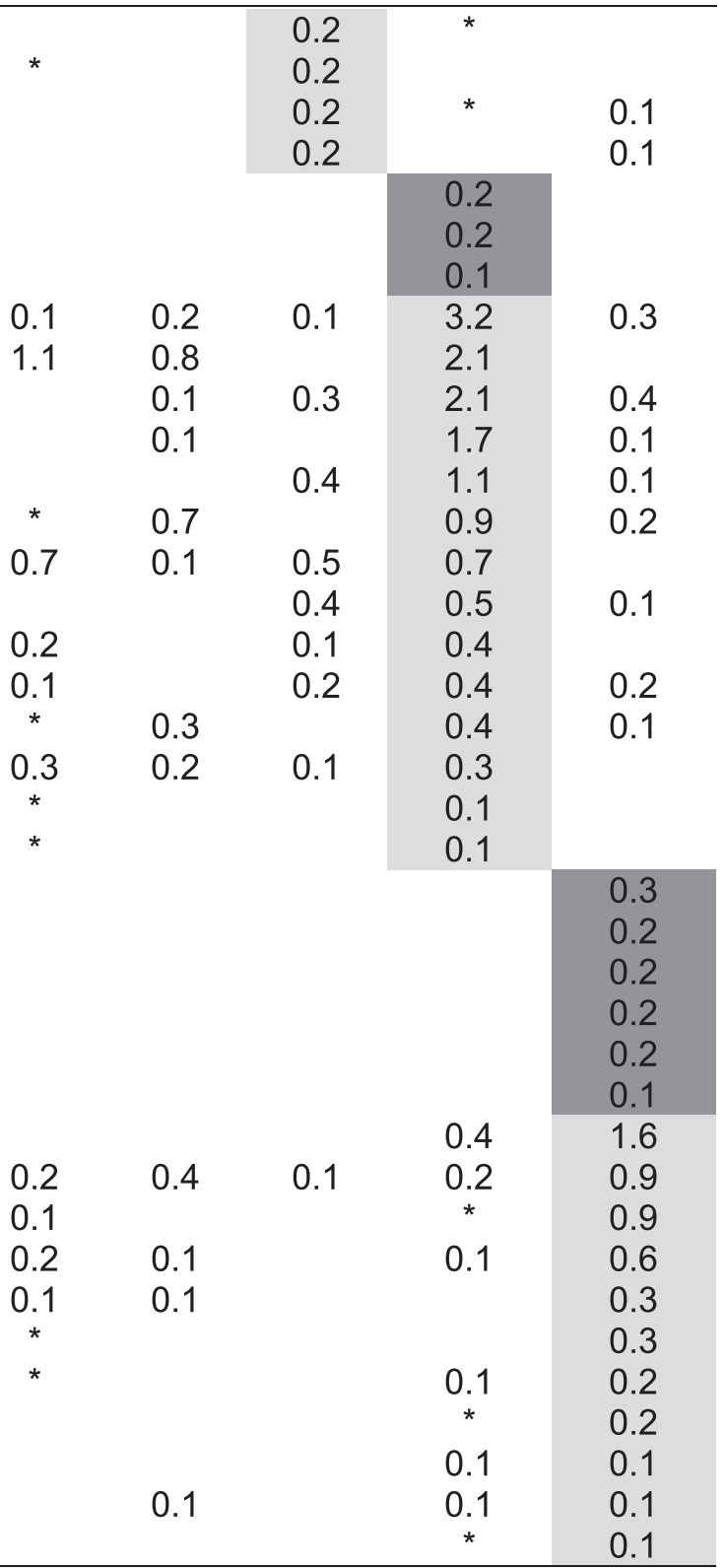

substrate preferences and bathymetric distribution for several species. Five major benthic molluskan communities were identified, being grain-size, gravel content, depth and hydrodynamic regime the abiotic

Table 5

Mollusks species richness in worldwide continental shelves.

\begin{tabular}{llcl}
\hline Locality & Latitude & $\begin{array}{l}\text { Species } \\
\text { richness }\end{array}$ & Reference \\
\hline Bay of Biscay (W France) & $46-47^{\circ} \mathrm{N}$ & 29 & Hily et al. (2008) \\
Enseñada S. Simon (NW Spain) & $42^{\circ} 18^{\prime} \mathrm{N}$ & 68 & Cacabelos et al. (2008) \\
Portugal & $36-42^{\circ} \mathrm{N}$ & 170 & This study \\
Southeastern Portugal & $36^{\circ} \mathrm{N}$ & 89 & Freitas et al. (2011) \\
Strait of Gibraltar (Spain/UK) & $35.5^{\circ} \mathrm{N}$ & 70 & Gofas (1998) \\
Alboran Sea (SE Spain) & $36.7^{\circ} \mathrm{N}$ & 162 & Rueda et al. (2009) \\
Uruguay & $34-36^{\circ} \mathrm{S}$ & 25 & Riestra et al. (2006) \\
Macaé (Rio de Janeiro-Brazil) & $22^{\circ} \mathrm{N}$ & 152 & Absalão et al. (2006) \\
Golfe d'Arguin (Mauritania) & $19-21^{\circ} \mathrm{N}$ & 118 & Michel et al. (2011) \\
\hline
\end{tabular}

factors best related to the molluskan data. This area is an important transitional zone, where elements of boreal, temperate and subtropical molluskan faunas coexist, particularly in the transitional faunal break zone, between Nazaré and S. Vicente canyons, in the western coast.

\section{Acknowledgments}

The first author benefited from a PhD grant (SFRH/BD/44231/2008) awarded by the Portuguese Foundation for Science and Technology (FCT - Fundação para a Ciência e Tecnologia) and co-financed by FSE (Fundo Social Europeu). This investigation was supported by the research projects "ACOSHELF” (POCI/MAR/56441/2004-PPCDT/MAR/ 56441/2004), "Monitorização Ambiental do Emissário Submarino e da ETAR da Guia do Sistema de Saneamento da Costa do Estoril", funded by SANEST, S.A. and "MeshAtlantic" (with the support of the European Union ERDF-Atlantic Area Program 2009-1/110). Colleagues from the Laboratory of Ecology and Biodiversity helped in the sampling and 
sorting of the material. Authors acknowledge the comments and suggestions of two anonymous reviewers.

\section{References}

Absalão, R.S., Moreira, J., Troncoso, J.S., 2006. Common environmental descriptors of two benthic amphi-Atlantic mollusc assemblages. Braz. J. Oceanogr. 54, 65-73.

Antoniadou, C., Koutsoubas, D., Chintiroglou, C., 2005. Mollusca fauna from infralittoral hard substrate assemblages in the North Aegean Sea. Belg. J. Zool. 135, 119-126.

Araújo, R., Bárbara, I., Sousa-Pinto, I., Quintino, V., 2005. Spatial variability of intertidal rocky shore assemblages in the northwest coast of Portugal. Estuar. Coast. Shelf Sci. 64, 658-670.

Ávila, S.P., Madeira, C., da Silva, C.M., Cachão, M., Landau, B., Quartau, R., Martins, A.M., 2008. Local disappearance of bivalves in the Azores during the last glaciation. J. Quat. Sci. 23, 777-785.

Benkendorfer, G., Soares-Gomes, A., 2009. Biogeography and biodiversity of gastropod molluscs from the eastern Brazilian continental shelf and slope. Lat. Am. J. Aquat. Res. 37, 143-159.

Bettencourt, A.M., Bricker, S.B., Ferreira, J.G., Franco, A., Marques, J.C., Melo, J.J., Nobre, A. Ramos, L., Reis, C.S., Salas, F., Silva, M.C., Simas, T., Wolff, W., 2004. Typology and Reference Conditions for Portuguese Transitional and Coastal Waters, Development of Guidelines for the Application of the European Union Water Framework Directive. INAG/IMAR, Lisboa, Portugal (100 pp.).

Bouchet, P., Gofas, S., 2013. World register of marine species. Accessed through http://www.marinespecies.org.

Byrnes, M.R., Hammer, R.M., Vittor, B.A., Kelley, S.W., Snyder, D.B., Côté, J.M., Ramsey, J.S. Thibaut, T.D., Phillips, N.W., Wood, J.D., Germano, J.D., 2003. Collection of environmental data within sand resource areas offshore North Carolina and the environmental implications of sand removal for coastal and beach restoration. OCS Report MMS 2000-056. U.S. Department of the Interior, Minerals Management Service, Leasing Division, Sand and Gravel Unit, Herndon, VA (256 pp.).

Cacabelos, E., Quintas, P., Troncoso, J.S., 2008. Spatial distribution of soft-bottom molluscs in the Ensenada de San Simón (NW Spain). Am. Malacol. Bull. 25, 9-19.

Clarke, K.R., Gorley, R.N., 2006. PRIMER v.6: User Manual/Tutorial PRIMER-E, 6 (Plymouth, England, 190 pp.).

Cunha, M.R., Paterson, G.L.J., Amaro, T., Blackbird, S., Stigter, H.C., Ferreira, C., Glover, A., Hilário, A., Kiriakoulakis, K., Neal, L., Ravara, A., Rodrigues, C.F., Tiago, A., Billett, D.S.M., 2011. Biodiversity of macrofaunal assemblages from three Portuguese submarine canyons (NE Atlantic). Deep-Sea Res. II 58, 2433-2447.

Cúrdia, J., Carvalho, S., Ravara, A., Gage, J.D., Rodrigues, A.M., Quintino, V., 2004. Deep macrofaunal assemblages from Nazaré Submanrine Canyon (N. W. Portugal). Sci. Mar. 68, 171-180.

Duff, M.L., Grall, J., Quiniou, L., 2009. First record of the gastropod Fusinus rostratus (Mollusca: Fasciolaridae) on the northern coast of Brittany (western Channel, France). Marine Biodiversity Records 2, e63.

Dajoz, R., 1971. Précis d'Ecologie. Ed. Dunod, Paris, France (549 pp.).

Fiúza, A.F.G., 1983. Upwelling patterns off Portugal. In: Suess, E., Thiede, J. (Eds.), Coastal Upwelling: Its Sediment Record. Plenum Publishing Corporation, New York, pp. 85-98.

Freitas, R., Ricardo, F., Pereira, F., Sampaio, L., Carvalho, S., Gaspar, M., Quintino, V., Rodrigues, A.M., 2011. Benthic habitat mapping: concerns using a combined approach (acoustic, sediment and biological data). Estuar. Coast. Shelf Sci. 92, 598-606.

Gaspar, M.B., Leitão, F., Santos, M.N., Chícharo, L., Dias, M.D., Chícharo, A., Monteiro, C.C., 2003. A comparison of direct macrofaunal mortality using three types of clam dredges. ICES J. Mar. Sci. 60, 733-742.

Gaspar, M.B., Sobral, M., Maia, F., Sobral, M.P., Viegas, M.C., Monteiro, C.C., 2004. A pescaria de moluscos bivalves na Zona Ocidental Norte e Zona Sul. Ponto de situação dos principais bancos (Setembro/Outubro 2002). Relatórios Científicos Técnicos IPIMAR. Série digital, 12 (33 pp.).

Gaspar, M.B., Sobral, M., Pereira, A., Maia, F., Sobral, M.P., Viegas, M.C., Monteiro, C.C., 2005. Prospecção dos bancos de moluscos bivalves nas Zonas Ocidental e Sul da costa portuguesa (campanha de pesca 2003). Relatórios Científicos Técnicos IPIMAR. Série digital, 22 (31 pp.).

Glémarec, M., 1973. The benthic communities of the European North Atlantic continental shelf. Oceanogr. Mar. Biol. Annu. Rev. 11, 263-289.

Gofas, S., 1998. Marine molluscs with a very restricted range in the Strait of Gibraltar. Divers. Distrib. 4, 255-266.

Graham, A., 1988. Molluscs: prosobranch and pyramidellid gastropods, Synopses of the British Fauna, 2nd ed. The Linean Society of London and The Estuarine and Brackish-water Sciences Association, Bath Press, Avon, UK (662 pp.).

Grall, J., Hall-Spencer, J.M., 2003. Problems facing maerl conservation in Brittany. Aquat. Conserv. Mar. Freshw. Ecosyst. 13, S55-S64.

Hidalgo, 1917. Fauna malacologica de España, Portugal y las Baleares. Moluscos testáceos marinos. In: Junta para Ampliación de Estudios e Investigaciones Centíficas (Ed.) Trabajos del Museo Nacional de Ciencias Naturales. Serie Zoologica, Núm. 30 (752 pp.).

Hily, C., 1987. Spatio-temporal variability of Chaetozone setosa (Malmgren) population on an organic gradient in the Bay of Brest (France). J. Exp. Mar. Biol. Ecol. 112, 201-216.

Hily, C., Le Loc'h, F., Grall, J., Glémarec, M., 2008. Soft bottom macrobenthic communities of North Biscay revisited: long-term evolution under fisheries-climate forcing. Estuar. Coast. Shelf Sci. 78, 413-425.

Huz, R., Lastra, M., López, J., 2002. The influence of sediment grain size on burrowing, growth and metabolism of Donax trunculus L. (Bivalvia: Donacidae). J. Sea Res. 47, 85-95.

Karakassis, I., Eleftheriou, A., 1997. The continental shelf of Crete: structure of macrobenthic communities. Mar. Ecol. Prog. Ser. 160, 185-196.
Koulouri, P., Dounas, C., Arvanitidis, C., Koutsoubas, D., Eleftheriou, A., 2006. Molluscan diversity along a Mediterranean soft bottom sublittoral ecotone. Sci. Mar. 70 (5), 573-583.

Krylova, E.M., 2006. Bivalves of seamounts of the north-eastern Atlantic. In: Mironov, A.N Gebruk, A.V., Southward, A.J. (Eds.), Biogeography of the North Atlantic Seamounts. KMK Scientific Press, Moscow, Russia, Moscow, pp. 76-95.

Legendre, L., Legendre, P., 1983. Numerical Ecology. Elsevier, Amsterdam, The Netherlands (853 pp.).

Macedo, M.C., Macedo, M.I., Borges, J., 1999. Conchas Marinhas de Portugal. Editora Verbo, Lisboa, Portugal (516 pp.).

Marques, V., 1987. A plataforma continental do Algarve: definição qualitativa das biocenoses de substrato móvel. , 31. Instituto Hidrográfico, Lisboa, Portugal (204pp.).

Martins, R., Azevedo, M.R., Mamede, R., Sousa, B., Freitas, R., Rocha, F., Quintino, V. Rodrigues, A.M., 2012. Sedimentary and geochemical characterization and provenance of the Portuguese continental shelf soft-bottom sediments. J. Mar. Syst. 91, 41-52.

Martins, R., Magalhães, L., Peter, A., San Martín, G., Rodrigues, A.M., Quintino, V., 2013a. Diversity, distribution and ecology of the family Syllidae (Annelida) in the Portuguese Coast (Western Iberian Peninsula). J. Helgol. Res. 67, 775-788.

Martins, R., Quintino, V., Rodrigues, A.M., 2013b. Diversity and spatial distribution patterns of the soft-bottom macrofauna communities on the Portuguese continental shelf J. Sea Res. 83, 173-186.

Martins, R., Sampaio, L., Rodrigues, A.M., Quintino, V., 2013c. Soft-bottom Portuguese continental shelf polychaetes: diversity and distribution. J. Mar. Syst. 213-214, 41-54.

Michel, J., Westphal, H., Cosel, R.V., 2011. The mollusk fauna of soft sediments from the tropical, upwelling-influenced shelf of Mauritânia (northwestern Africa). Palaios 26, $447-460$.

Monegatti, P., Raffi, S., 2007. Mediterranean-middle eastern Atlantic façade: molluscan biogeography and ecobiostratigraphy throughout the Late Neogene. In: Ávila, S.P., de Frias Martins, A.M. (Eds.), Proceedings of the 1st Atlantic Islands Neogene. International Congress. Açoreana, Ponta Delgada, pp. 126-139.

Moreira, M.H., Queiroga, H., Machado, M.M., Cunha, M.R., 1993. Environmental gradients in a southern Europe estuarine system: Ria de Aveiro, Portugal. Implications for soft bottom macrofaunal colonization. Neth. J. Aquat. Ecol. 27, 465-482.

Moulaert, I., Hostens, K., Hillewaert, H., Wittoeck, J., 2007. Spatial variation of the macrobenthos species and communities of the Belgian continental shelf and the relation to environmental variation. ICES A 09, 1-13.

Narayanaswamy, B.E., Renaud, P.E., Duineveld, G.C.A., Berge, J., Lavaleye, M.S.S., Reiss, H., Brattegard, T., 2010. Biodiversity trends along the Western European Margin. PLoS ONE 5 (12), e14295.

Nascimento, S., Franco, P., Sousa, F., Dias, J., Neves, F., 2012. Automated computational delimitation of SST upwelling areas using fuzzy clustering. Comput. Geosci. 43 207-216.

Oliveira, A., Santos, A.I., Rodrigues, A., Vitorino, J., 2007. Sedimentary particle distribution and dynamics on the Nazaré canyon system and adjacent shelf (Portugal). Mar. Geol. 246 $105-122$.

Pereira, S.G., Lima, F.P., Queiroz, N.C., Ribeiro, P.A., Santos, A.M., 2006. Biogeographic patterns of intertidal macroinvertebrates and their association with macroalgae distribution along the Portuguese coast. Hydrobiologia 555, 185-192.

Pereira, A., Palanco, I., Rufini, M., Moreno, O., Gaspar, M.B., 2007. La pesca de bivalvos en el litoral oceanico de la costa sur-occidental de la Península Ibérica: Descriptión de la pesquería y artes de pesca, medidas de gestión, biología de las especies comerciales y catálogo de especies de bivalvos. Junta de Andalucía, Instituto de Investigación y Formación agraria y Pesquera, Consejería de Innovación, Ciencia y Empresa, Consejeria de Agricultura y Pesca (141 pp.).

Petersen, C.G.J., 1918. The sea bottom and its production of fishfood. A survey of the work done in connection with valuation of the Denmark waters from 1883-1917. Report of the Danish Biological Station, 25, pp. 1-62.

Picard, J., 1965. Recherches qualitatives sur les biocoenoses marines des substrats meubles dragables de la région Marseillaise. Recueil Trav. Station Mar. Endoume $52,1-160$.

Prena, J., Schwinghamer, P., Rowell, T.W., Gordon, D.C., Golkinson, K.D., Vass, W.P., McKeown, D.L., 1999. Effects of experimental otter trawling on the macrofauna of a sandy bottom ecosystem on the Grand Banks of Newfoundland: analysis of trawl bycatch and effects on epifauna. Mar. Ecol. Prog. Ser. 181, 107-124.

Quintino, V., Rodrigues, A.M., Gentil, F., 1986. Etude faunistique et coenotique des Molusques (Bivalves et Gastéropodes) des lagunes Obidos et Albufeira (Portugal). Haliotis 15, 83-90.

Quintino, V., Gentil, F., Rodrigues, A.M., Peneda, M.C., 1987. Macrozoobenthic community structure in the lagoon of Albufeira, western coast of Portugal. J. Exp. Mar. Biol. Ecol. 106, 229-241.

Quintino, V., Rodrigues, A.M., Gentil, F., 1989. Assessment of macrozoobenthic communities in the lagoon of Óbidos, western coast of Portugal. Sci. Mar. 53, 645-654.

Raffi, S., Stanley, S., Marasti, R., 1985. Biogeographic patterns and Plio-Pleistocene extinction of Bivalvia in the Mediterranean and southern North Sea. Paleobiology 11 (4), 368-388.

Retière, C., 1979. Contribution à la connaissance des peuplements benthiques du golfe Normano-Breton. Université Rennes I, France (Thèse d'Etat, 431 pp.).

Riera, R., Delgado, J., Rodríguez, M., Monterroso, O., Ramos, E., 2012. Macrofaunal communities of threatened subtidal maërl seabeds on Tenerife (Canary Islands, north-east Atlantic Ocean) in summer. Acta Oceanol. Sin. 31, 98-105.

Riestra, G., Lozoya, J.P., Fabiano, G., Santana, O., Carrizo, D., 2006. Benthic macroinvertebrate bycatch in the snail Zidona dufresnei (Donovan) fishery from the Uruguayan continental shelf. Pan-Am. J. Aquat. Sci. 1, 104-113.

Rodrigues, A.M., Quintino, V., 1993. Horizontal biosedimentary gradients across the Sado estuary, Western coast of Portugal. Neth. J. Aquat. Ecol. 27, 449-464. 
Rodrigues, A.M., Meireles, S., Pereira, T., Gama, A., Quintino, V., 2006. Spatial patterns of benthic macroinvertebrates in intertidal areas of a southern European estuary: The Tagus, Portugal. Hydrobiologia 555, 99-113.

Rodrigues, A.M., Quintino, V., Sampaio, L., Freitas, R., Neves, R., 2011. Benthic biodiversity patterns in Ria de Aveiro, Western Portugal: environmental-biological relationships. Estuar. Coast. Shelf Sci. 95, 338-348.

Rodríguez, R.G., Sanchéz, J.M.P., 1997. Moluscos Bivalvos de Canarias. Ediciones del Cabildo Insular de Gran Canaria, Las Palmas de Gran Canaria (425 pp.).

Rueda, J., Gofas, S., Urra, J., Salas, C., 2009. A highly diverse molluscan assemblage associated with eelgrass beds (Zostera marina L.) in the Alboran Sea: microhabitat preference, feeding guilds and biogeographical distribution. Sci. Mar. 73 (4), 679-700.

Rufino, M.M., Gaspar, M.B., Maynou, F., Monteiro, C.C., 2008. Regional and temporal changes in bivalve diversity off the south coast of Portugal. Estuar. Coast. Shelf Sci. 80, 517-528.

Rufino, M.M., Gaspar, M.B., Ferreira, A.M., Maynou, F., Monteiro, C.C., 2010. Ecology of megabenthic bivalve communities from sandy beaches on the south coast of Portugal. Sci. Mar. 74 (1), 163-178.

Saldanha, 1995. Fauna Marinha Atlântica. Publicações Europa-America. Mem-Martins, Portugal (364 pp.).

Silva, C.M., Landau, B., Domènech, R., Martinell, J., 2006. Pliocene Atlanto-Mediterranean biogeography of Patella pellucida (Gastropoda, Patellidae): Palaeoceanographic implications. Palaeogeogr. Palaeoclimatol. Palaeoecol. 233, 225-234.
Silva, C.M., Landau, B., Domènech, R., Martinell, J., 2010. Pliocene Atlantic molluscan assemblages from the Mondego Basin (Portugal): age and Palaeoceanographic implications. Palaeogeogr. Palaeoclimatol. Palaeoecol. 285, 248-254.

Spalding, M., Fox, H., Allen, G.R., Davidson, N., Ferdana, Z.A., Finlayson, M., Halpern, B.S Jorge, M.A., Lombana, J.A., Lourie, S.A., Martin, K.D., Mcmanus, E., Molnar, J., Recchia, C.A., Robertson, J., 2007. Marine ecoregions of the world: a bioregionalisation of coastal and shelf areas. Bioscience 57, 573-583.

Tebble, N. 1976. British bivalve seashells, A Handbook for Identification2nd ed. British Museum (Natural History), Alden Press, Oxford, UK (212 pp.).

Thompson, T.E., 1988. Molluscs: benthic opisthobranchs: Mollusca, Gastropoda: keys and notes for the identification of the species, Synopses of the British Fauna2nd ed. The Linean Society of London and The Estuarine and Brackish-water Sciences Association, Bath Press, Avon, UK (356 pp.).

Thorson, G., 1957. Bottom communities (sublittoral or shallow shelf). In: Hedgpeth, J.W. (Ed.), Treatise on Marine Ecology and Paleoecology. Geological Society of America, USA, pp. 461-534.

Urra, J., Ramírez, A.M., Marina, P., Salas, C., Gofas, S., Rueda, J., 2013. Highly diverse molluscan assemblages of Posidonia oceanica meadows in northwestern Alboran Sea (W Mediterranean): seasonal dynamics and environmental drivers. Estuar. Coast. Shelf Sci. $117,136-147$

Zenetos, A., 1996. Classification and interpretation of the established Mediterranean biocoenoses based solely on bivalve molluscs. J. Mar. Biol. Assoc. U. K. 76, 403-416. 